\title{
Is there an interaction between perceived direction and perceived aspect ratio in stereoscopic vision?
}

\author{
RAYMOND VAN EE \\ University of California, Berkeley, Califormia \\ and \\ CASPER J. ERKELENS \\ Helmholtz Institute, Utrecht, The Netherlands
}

\begin{abstract}
In monocular vision, the horizontal/vertical aspect ratio (shape) of a frontoparallel rectangle can be based on the comparison of the perceived directions of the rectangle's edges. In binocular vision of a typical three-dimensional scene (when occlusions are present), this is not the case: Frontoparallel rectangles would be perceived in a distorted fashion if an observer were to base perceived aspect ratio on the perceived directions of the rectangle's edges. We psychophysically investigated stereoscopically perceived aspect ratios of frontoparallel occluding and occluded rectangles for various distances and fixation depths. We found that observers did not perceive the distortions that would be predicted on the basis of the above-mentioned comparison of the perceived visual directions of the edges of the rectangle. Our results strongly suggest that the mechanism that determines perceived aspect ratio is dissociated from the mechanism that determines perceived direction. The consequences of the findings for the Kanizsa, Poggendorff, and horizontal/vertical illusions are discussed.
\end{abstract}

In stereoscopic vision research, we are interested in how the three-dimensional (3-D) layout of a scene is perceived. Both perceived direction and perceived horizontal/vertical aspect ratio (or shape) play an important role in the recovery of the 3-D layout. Recently, Erkelens, Muijs, and van Ee (1996) studied the perceived direction of two stimuli relative to each other in the neighborhood of occluders. The authors' results explicitly indicated that the interaction between perceived direction and perceived aspect ratio was an unresolved topic, because the existing standard model of stereoscopic vision did not relate the two in a satisfying fashion.

Erkelens et al. (1996) instructed observers to align a stereoscopically visible slider with the rim of a stereoscopically visible frontoparallel circular disk (Figure 1) so that the perceived visual directions of both the slider and the rim were identical. The disk was hovering in front of a background, and the slider was located in the background. At the leftmost part of the rim, observers positioned a

R.v.E. was supported by the Foundation for Life Sciences (SLW Talent Stipendium, NWO 810-404-006/1) of the Netherlands Organization for Scientific Research. R.v.E was also supported by Grant RG-34/96 from the Human Frontier Science Project (to M. S. Banks). We thank $\mathrm{H}$. Ono for many discussions concerning conceptualization and interpretation of this study. We also thank B. L. Anderson, M. K. Banks, J. S. Lappin, S. Ohtsuka, and J. M. Wolfe for comments or discussions. We are grateful to S. M. McNab for linguistic advice. Correspondence concerning this article should be addressed to $\mathrm{R}$. van $\mathrm{Ee}$, Helmholtz Institute, Princetonplein 5, 3584 CC Utrecht, The Netherlands. vertical slider as if the slider was only viewed by the left eye. At the rightmost part of the rim, a vertical slider was aligned as if it was only viewed by the right eye. Figure 1B provides an impression of the finding of Erkelens et al. At the top and bottom parts of the rim, observers positioned a horizontal slider as if the slider was viewed from a vantage point at eye level. In other words, the aspect ratio of the circular occluder and the aspect ratio of the occluded region (as enclosed by the set of slider alignments) were not identical. Nevertheless, the observers were of the opinion that they perceived an undeformed circular occluder and that the occluded region was also circular. Thus, there appears to be a discrepancy between the subjectively perceived aspect ratio of the occluded region and the objective aspect ratio of the occluded region deduced from the alignment of the slider. We will refer to the latter, objectively deduced horizontal/vertical aspect ratio based on alignment as the alignment-enclosed aspect ratio.

How large is the discrepancy between the (subjectively) perceived aspect ratio of the occluded region and the (objective) alignment-enclosed aspect ratio? Using Figure 2, predicting the alignment-enclosed horizontal dimension of the occlusion for frontoparallel planes is straightforward. In this figure, line $A B$ is occluded by line $A^{\prime} B^{\prime}$. A and $B$ are only visible monocularly. $A$ is visible only with the left eye. Alignment of A with another target is performed as if both targets were viewed with only the left eye (Erkelens et al., 1996). B is visible only with the right eye. Alignment of $B$ with another target is performed as if 


\section{(A) Task}

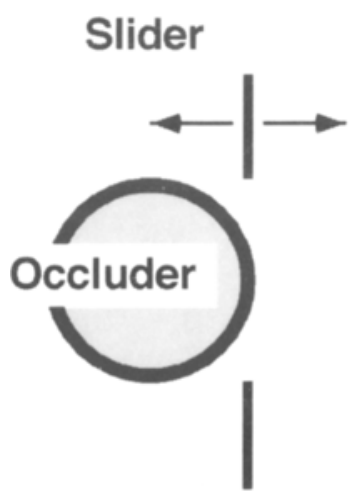

(B) 3D View of Result

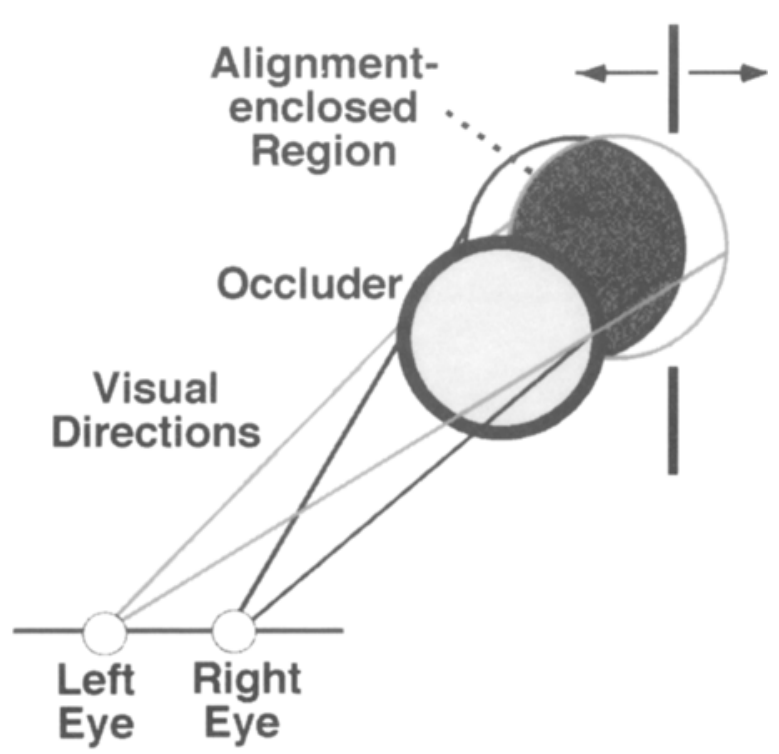

\begin{abstract}
Figure 1. Task and results according to Erkelens, Muijs, and van Ee (1996). (A) Schematic view of the subject's perception of the scene in the experiment of Erkelens et al. An occluding disk was hovering in front of a background. In fact, the occluder was monocularly invisible because it was presented as a random-dot pattern relative to an identical background random-dot pattern (Julesz stereogram). A binocularly visible slider was presented in the background, and the task of the subject was to align the slider with the rightmost (or leftmost) part of the disk's rim. One slider was visible at a time. The slider consisted of two parts that were segments of an (invisible) line. The slider was oriented either vertically (shown at the right side of the rim) or horizontally (not shown). (B) An artist's conception of the results of Erkelens et al. The figure shows a slider when it was perceived to be aligned with the rightmost part of the disk's rim. Erkelens et al. found that the horizontal dimension of the occlusion (in fact, the dark area in the background constituted by the slider alignments at the leftmost and rightmost parts of the occluder's rim) is smaller than the vertical dimension of the occlusion.
\end{abstract}

both targets were viewed with only the right eye (Erkelens et al., 1996). For the moment, let us assume that the visual system determines perceived aspect ratio on the basis of low-level visual information that is detected by the retinas. All retinal information that is available to measure horizontal and vertical dimensions consists of visual angles. The visual angle between the fovea and the retinal projection of a target is called the local sign. If an observer were to use the retinal local signs (angles $\alpha$ and $\beta$ ) directly to estimate the horizontal dimension that is occluded in the background $(\gamma)$, he or she would overestimate this dimension by an amount equal to the disparity $(\delta)$ between the fixation plane and the background:

$$
\begin{gathered}
\mathrm{A}^{\prime} \mathrm{B}^{\prime}=\alpha+\beta=\gamma+\delta, \\
\gamma=\alpha+\beta-\delta .
\end{gathered}
$$

Expressed in words, the difference between the horizontal angular dimension of a frontal occluder and the alignmentenclosed horizontal angular dimension of its occlusion is equal to the difference in the disparity between the fixation plane and the background. If the difference in dispar- ity is $\delta$ and the horizontal dimension of the foreground plane is $S$, the horizontal angular dimension ratio of background/foreground equals

$$
\frac{\mathrm{S}-\delta}{S}
$$

For example, if the difference in disparity is $0.5^{\circ}$ and the horizontal dimension of the foreground plane is $5.2^{\circ}$ (as it will be in our experiments), the horizontal angular dimension ratio of background $/$ foreground $=4.7 / 5.2=0.9$. Because the eyes are oriented horizontally next to each other, no such misestimation is predicted in the vertical direction. If the perceived dimension of the occluder in this example were identical to the alignment-enclosed dimension of its occlusion (the perceived visual directions along the rim), one would expect that a rectangle whose vertical dimension is $9.6 \%$ smaller than its horizontal dimension would be perceived as a square. ${ }^{l}$ Note that it is not only distortion that is predicted, but also discontinuity in the visual direction of the background at the transition from monocular to binocular regions (van Ee, Banks, \& Backus, 1999). 


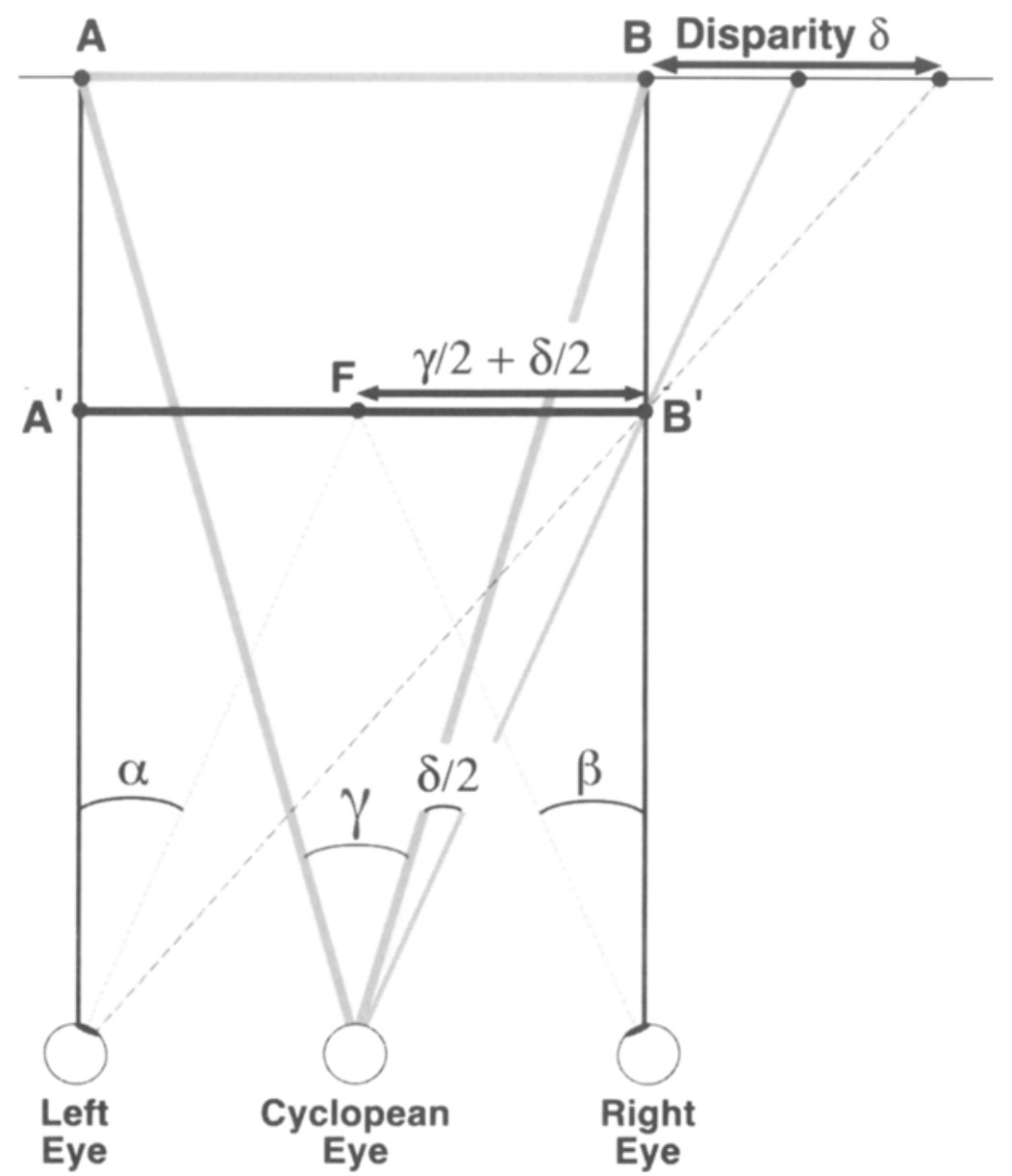

Figure 2. The perceived horizontal dimension difference between two distinct depth planes: Schematic top view of an observer fixating point $F$ in the plane of line $A^{\prime} B^{\prime}$. This line occludes line $A B$. A and $B$ are only visible monocularly. For the observer, $A$ looks as if it is aligned with $A^{\prime}$, and $B$ looks as if it is aligned with $B^{\prime}$ (Erkelens, Muijs, $\&$ van $\mathrm{Ee}, 1996)$. If the observer were to use the retinal local signs ( $\alpha$ and $\beta$ ) directly to estimate the dimension that is occluded in the background $(\gamma)$, he or she would overestimate this dimension by an amount that is equal to the disparity between the fixation plane and the background. Note that this statement is true for fixation in any depth plane. If fixation is behind the plane of interest, the sign of the disparity is reversed. From an operational point of view, it is convenient to use a single vantage point (the so-called cyclopean eye) from which the stereoscopic visual world is viewed, rather than using the views of two eyes. This cyclopean eye is located midway on the line that connects the nodal points of the eyes. But note that, mathematically speaking, the location of the single vantage point is essentially irrelevant for the above-derived statements.

Figure 3 demonstrates, in the form of stereograms, the discrepancy between the subjectively perceived aspect ratio of the occluded region and the objective alignmentenclosed aspect ratio. ${ }^{2}$ The two stereograms in Figure 3 consist of a background chessboard pattern and a foreground feature that is hovering in front of the background. Figure $3 \mathrm{~A}$ addresses the perceived aspect ratio of the foreground. In Figure 3A, observers perceive the sliders to be aligned with the rim of the occluding disk; that is, the perceived visual directions of both the slider and the part of the rim nearest to the slider are identical. Closer examination of the half-images of the stereogram shows that, in fact, the left slider is physically aligned with the leftmost part of the rim only in the left eye's half-image of the stereogram; the right slider is physically aligned with the rightmost part of the rim only in the right eye's half-image. Observers perceive the number of chessboard elements subtended by the occluding disk to be eight in the horizontal direction and nine in the vertical direction, which means that the number of elements in the vertical direction is $12.5 \%$ larger than that in the horizontal direction. This significant difference is not reflected in the perceived aspect ratio because both the aspect ratio of the occluder and the aspect ratio of the occlusion are perceived as being 


\section{(A) Occluder Shape}
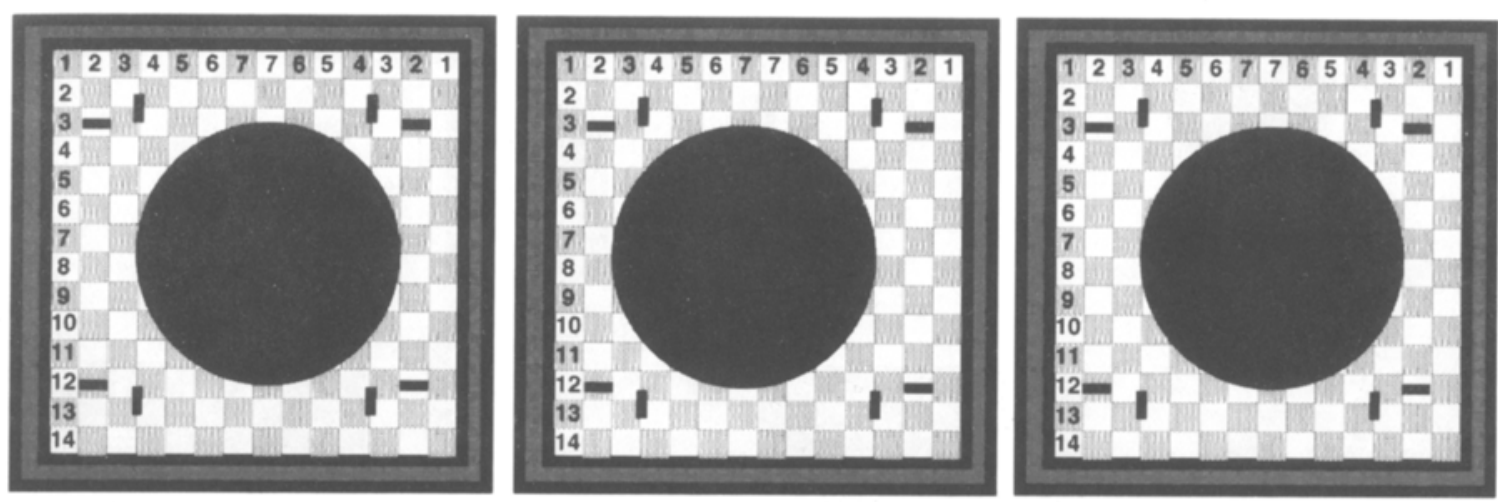

(B) Background Shape Uncrossed Crossed
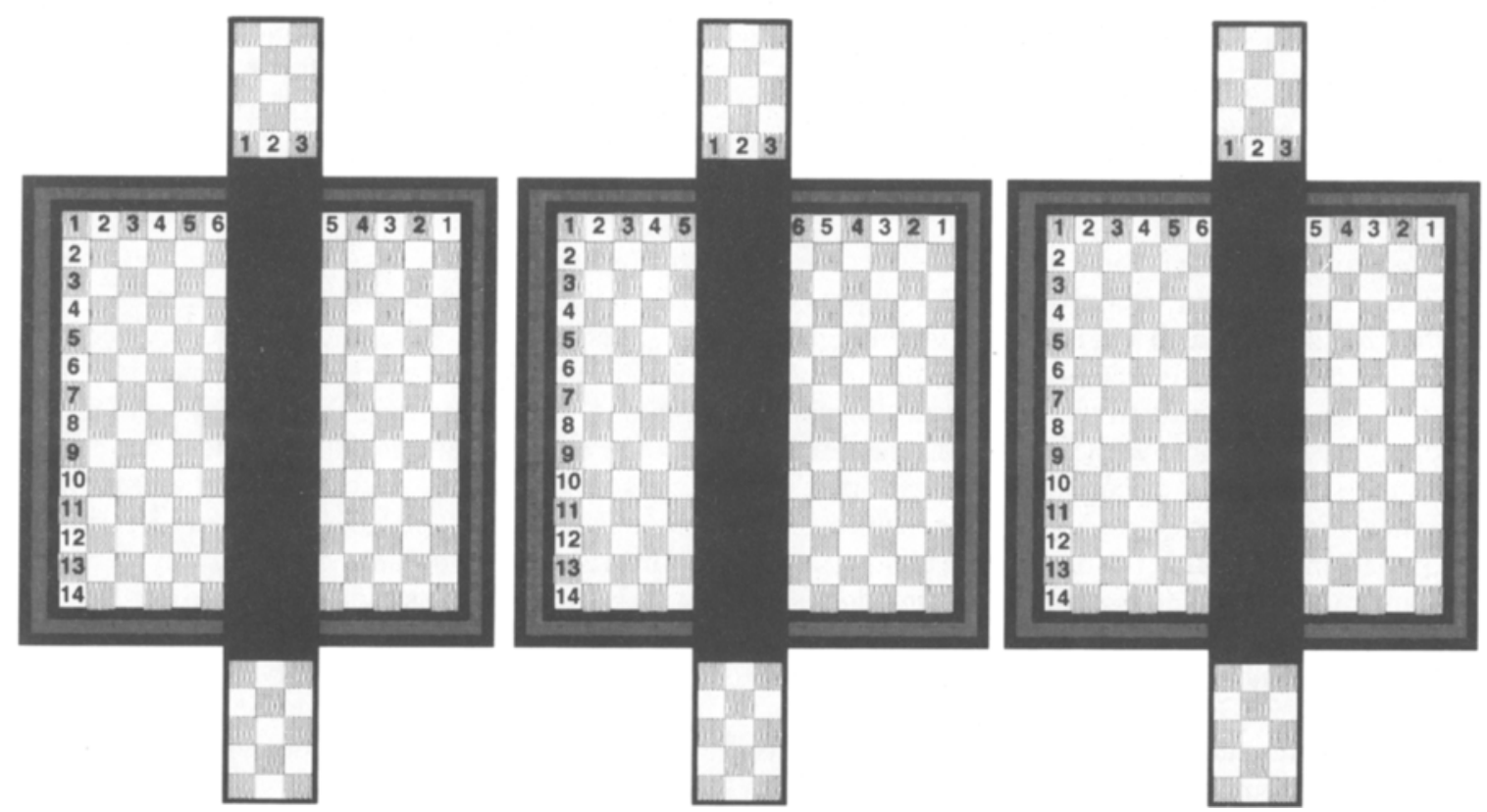

Figure 3. Discrepancy between perceived alignment and perceived aspect ratio. (A) This demonstration of the results obtained by Erkelens, Muijs, and van Ee (1996) shows that there is a discrepancy between perceived alignment and perceived horizontal/vertical aspect ratio of the occluder. In each of the half-images, four sliders are presented: two vertical and two horizontal sliders. In this particular example, observers perceive the sliders to be aligned with the corresponding rim of the occluding disk (after they have stable fusion of the two half-images). Closer examination of the half-images of the stereogram shows that, in fact, the left slider is physically aligned with the leftmost part of the rim in the left eye's half-image of the stereogram; the right slider is physically aligned with the rightmost part of the rim in the right eye's half-image. When observers are asked to count the number of chessboard elements subtended by the occluding disk (again, after they have fused the two half-images), they count eight elements in the horizontal direction and nine in the vertical direction, which means that the number of elements in the vertical direction is $12.5 \%$ larger than that in the horizontal direction. This significant difference is not reflected in the perceived shape, because the shape of both the occluder and the occlusion is perceived as being circular or only slightly elongated in the vertical direction. In the study by Erkelens et al., the occluding disk was monocularly indistinguishable from the background pattern (see the caption of Figure 1A). (B) Most observers report that the shape of the background feature is a square or a standing rectangle (with the vertical dimension slightly larger than the horizontal dimension). However, when the observers count the number of chessboard elements (after they have stable fusion of the two half-images), they find out that the number of elements in the horizontal direction is larger than that in the vertical direction; they perceive six elements on either side of the bar. Given that the bar is three elements wide, the number of elements in the horizontal direction (15) is now $7 \%$ larger than that in the vertical direction (14). Again, this significant difference is not reflected in the perceived shape. 

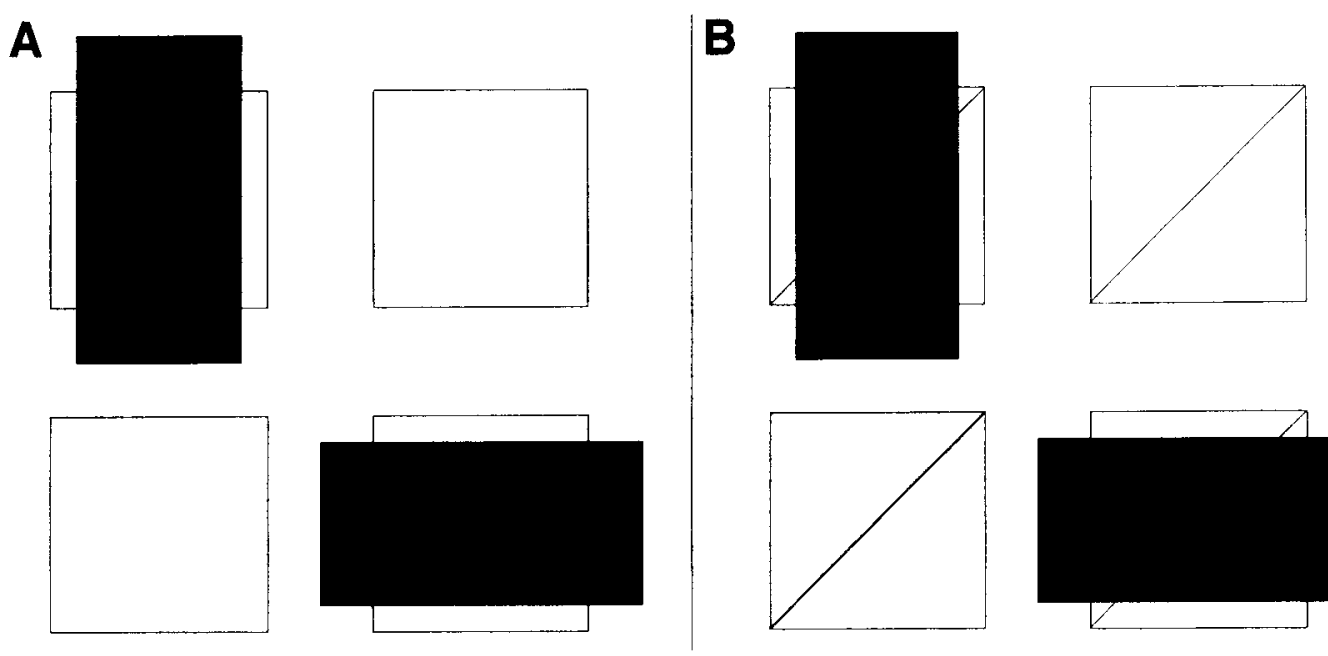

Figure 4. The Kanisza and Poggendorff illusions in two-dimensional drawings. (A) Kanizsa's compressed square illusion. The occluded square in the top-left corner looks more compressed in the horizontal direction than do the nonoccluded squares of the same size. The top-left configuration is the one that is generally known as Kanizsa's square. The bottom-right configuration shows that after $90^{\circ}$ of rotation, the illusion is just as strong, but now in the vertical direction. (B) Poggendorff's shifted line segment illusion. The two lines that are present on either side of the rectangle are, in fact, segments of one line. But, in the top-left square, it looks as if the right segment is shifted upward relative to the left segment. The top-left configuration is the one that is generally known as the Poggendorff illusion. The bottom-right configuration shows that after $90^{\circ}$ of rotation, the illusion is also strong, but now in the horizontal direction.

equal to unity (circular). Figure 3B addresses the perceived aspect ratio of the background. Most observers report that the shape of the background feature in Figure 3B is a square or a standing rectangle (with the vertical dimension slightly larger than the horizontal dimension). However, observers perceive the number of chessboard elements in the horizontal direction to be larger than that in the vertical direction; they perceive six elements on either side of the bar. Given that the bar is three elements wide, the number of elements in the horizontal direction is now $7 \%$ larger than that in the vertical direction. This significant difference is not reflected in the perceived aspect ratio of the chessboard pattern in the background.

The results mentioned so far can be understood if one assumes that alignment in a monocular region is equivalent to equating the local signs (visual directions) in the eye that can see the monocular region caused by the occluder (Erkelens \& van de Grind, 1994). ${ }^{3}$

Support for the findings of Erkelens et al. (1996) comes from reports of Lillakas, Ono, and Grove (1998), Ohtsuka and Ono (1998), and Suzuki, Segal, Lillakas, and Ono (1998). Suzuki et al. and Lillakas et al. examined background distortions by having subjects read text on a computer screen that was partly occluded by a vertical rod. They found that the monocular areas were readable but that visual directions were perceptually displaced. However, they reported that the perceived aspect ratio of the letters and words were not deformed. Ohtsuka and Ono investigated both Kanizsa's compressed square illusion and the Poggendorff illusion in stereoscopic viewing. Figure 4 shows two-dimensional (2-D) examples of these il- lusions. In both illusions, Ohtsuka and Ono confirmed that alignment near the monocular areas caused by the occluders was based on visual information provided by the eye to which the monocular area was visible. In the Discussion section, we will address these illusions in more detail.

The above-mentioned observations are interesting because they are not described by the existing standard model of the perception of binocular direction. The standard model, formulated originally by Alhazen, Wells, and Hering and consolidated by Ono and others (for a review, see Howard \& Rogers, 1995; Schor, 1999), states that the visual directions derived from the two eyes images will be perceived as if the observer were viewing the scene from a single vantage point midway between the two eyes; this point is called the cyclopean eye (Figure 2). Modern, comprehensive, and extended versions of the standard model are presented by Banks et al. (1997), Ono and Mapp (1995), and van de Grind, Erkelens, and Laan (1995). ${ }^{4}$ The cyclopean eye (like either real eye) has a 2-D structure on which each location represents a visual direction. According to the standard model, all visual targets viewed by either eye are included in the cyclopean eye and, thus, are perceived in binocular vision.

It is impossible to fit the complete set of visual directions into a 2-D cyclopean eye when there are occluders in the visual field, because one eye will contain a number of projections of visual targets that are not visible to the other eye (Anderson \& Nakayama, 1994; Erkelens \& van de Grind, 1994). Similarly, it is impossible to paint a 3-D picture on canvas, which has been known since the time of Leonardo da Vinci, who was one of the first to make this 
explicit. The cyclopean eye has no room for a greater number of projected targets than the number of projected targets in either of the eyes. Thus, the above-mentioned observations, in which both the background and the foreground are (at least subjectively) perceived without distortion, are not compatible with the standard model of binocular perception of direction.

\section{MOTIVATION FOR NEW EXPERIMENTS}

From existing experimental work, it is impossible to derive systematic characteristics of the objectively perceived aspect ratio of both occluders and occluded objects in stereoscopic vision. We will describe the results of four experiments. In the first experiment, we psychophysically investigated the impression, as reported by the observers of Erkelens et al. (1996), that one perceives an undeformed occluder. Figure 2 shows that the fixation depth has theoretically a profound influence on perceived aspect ratio if this ratio is based on local sign information (see also Banks et al., 1997). The magnitude of this influence is significant because it is of the same order as the disparity. Therefore, in Experiment 2, we investigated the influence of fixation depth on the perceived aspect ratio of an occluder. The observers in the study of Erkelens et al. reported that they perceived an undeformed occluded object. Therefore, in the third and fourth experiments, we systematically investigated whether an occluder distorts the perceptual space behind it.

\section{GENERAL METHOD}

\section{Subjects}

Fifteen subjects participated in the four experiments. They were checked for normal stereo vision by means of partially decorrelated Julesz random-dot test images. They were naive with respect to the purposes of the experiments. Five subjects were experienced in stereoscopic experiments. The other subjects were inexperienced. Nine subjects showed refraction anomalies, which were corrected by their own contact lenses or glasses. Eight subjects participated in each of the four experiments.

\section{Apparatus}

The apparatus has been described previously (van Ee \& Erkelens, 1995). The subject viewed stereograms that were generated by an HP 750 graphics computer. Subsequently, the stimuli were backprojected onto a large frontoparallel translucent screen by a projection TV (Barco Data 800). The size of the screen was $70^{\circ} \times 70^{\circ}$. The subjects were seated in front of the screen at a distance of $1.5 \mathrm{~m}$. Head movements were restricted by a chinrest and a skullrest. The interocular axis was both horizontal and parallel to the screen. The left and right eyes' half-images of the stereogram were shown in red and green, respectively, and both were presented in each trial afresh at a frequency of $70 \mathrm{~Hz}$. The subjects wore anaglyph glasses. The left and right eyes viewed the screen through filters matched to the emission spectra of the red and green phosphors of the TV, respectively; no crosstalk was observed. The relative brightness of both the red and the green half-images was adjusted to look equally bright when viewed through the glasses.

Because the stimuli were large random-dot patterns, which consequently illuminated the surroundings, it was impossible to work in a completely dark room. In order to make uncontrolled features in the room, such as furniture, impossible to see, we used black cloths that were attached between the side-edges of the screen and the headrest. There was black carpet on the floor. In order to make the lighting conditions in the room more similar across trials (which were relatively bright when the large background was presented, as compared with trials in which only the rectangle was shown), we dimly illuminated the ceiling of the room.

\section{Stimuli}

In all of the experiments, we presented frontoparallel rectangular stimuli (Figures 5 and 10), of which the vertical dimension relative to the horizontal dimension had to be judged both in the presence and in the absence of an occluding bar in front of the rectangle. All dot sizes were $3.0 \times 3.0$ arcmin ( 1 pixel).

The shift between the red and the green half-images of both the rectangles and the foreground bar was modified independently so that the rectangles were perceived at different depths in front of the background. We will call this shift on the screen disparity. The background was always presented in the plane of the screen, so that there was no disparity between its half-images. All of the disparities in this paper will be crossed disparities. Whenever there was disparity present, the left eye's half-image was shifted to the right over half the disparity; the right eye's half-image was shifted to the left by the same magnitude. This means that the eccentricity of the center of the rectangle, relative to the head, was unaffected by the disparity.

The display duration was fixed (except for Experiment 1B) at $2.0 \mathrm{sec}$. The period was kept short so that it was impossible to perform the task by counting both the number of random dots and the gaps between them. Except for Experiment 2, the subjects were free to make eye movements while viewing the stereogram. The blanking period between stimuli was $300 \mathrm{msec}$.

\section{Task, Procedure, and Data Analysis}

In all of the following experiments, the subjects judged the hor$\mathrm{izontal} / \mathrm{vertical}$ aspect ratio of a rectangle. The aspect ratio of the presented rectangles differed between $86 \%$ and $114 \%$. There were 11 different aspect ratios presented, which were taken from the following set: $0.86,0.92,0.96,0.98,0.99,1.00,1.01,1.02,1.04,1.08$, or 1.14. Each trial was repeated 12 times randomly intermixed with the other aspect ratios. Thus, a particular condition consisted of 132 $(11 \times 12)$ trials. No feedback was given about the results.

Psychometric functions were fitted to the data in order to determine the aspect ratio at which both, alternative answers were given equally often (known as the $50 \%$ point). For 3 subjects, the standard deviation in the $50 \%$ point across trials was determined by repeating the experiment three times; for the rest of the subjects, it was determined by a standard Monte Carlo simulation. These standard deviations across trials within a subject were small: about $0.3 \%$. The subjects showed identical trends in their data. Therefore, we will present the averaged data across subjects. In all of the graphs, error bars represent the standard deviation of the data across 8 subjects. ${ }^{5}$

\section{EXPERIMENT 1 \\ Perceived Aspect Ratio of Rectangular Stimuli}

In this introductory experiment, we investigated the influence of the disparity of the rectangle (Experiment $1 \mathrm{~A}$ ) and the display duration (Experiment 1B) on the perceived aspect ratio.

\section{Method}

Figure 5A shows a schematic drawing of the stimulus. The stimuli were essentially traditional Julesz random-dot stereograms, which 
(A) Experiments 1a and 1b

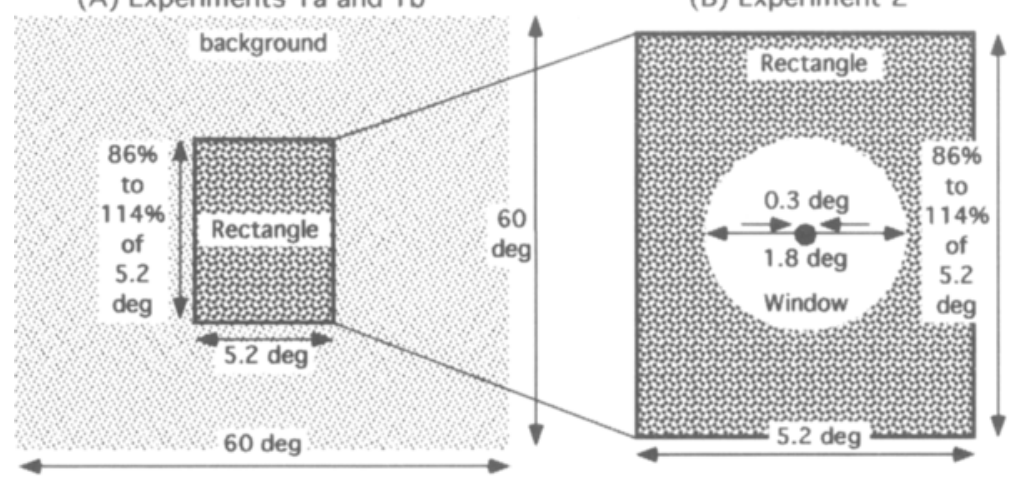

(C) Experiment 3

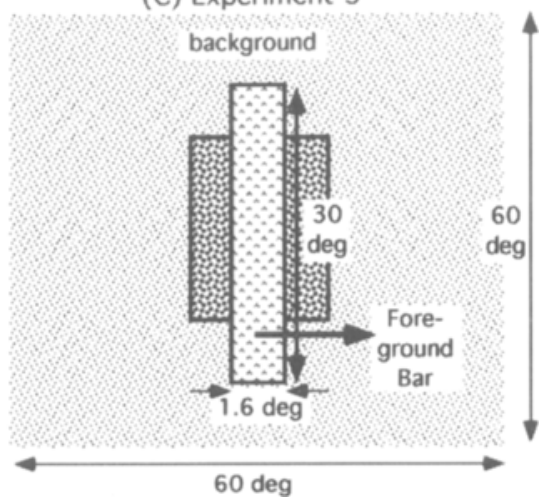

Figure 5. Julesz random-dot stimuli used in Experiments 1-3. We presented frontoparallel rectangular stimuli in the center of the screen. In the experiments, the background $(A, C)$, the rectangle $(A, B, C)$, and the foreground bar $(C)$ contained identical randomdot textures, so they were monocularly indistinguishable. All dot sizes were $3 \times 3$ arcmin. The monocularly visible contours (width, 6 arcmin; drawn in black) around the rectangle $(A, B, C)$ were only visible in particular conditions. The background $\left(60^{\circ} \times 60^{\circ}\right)$ was also only visible in particular conditions; its disparity was always $0^{\circ}$. (A) Schematic drawing of the stimulus used in Experiment 1 . The disparity of the rectangle was $0.5^{\circ}, 1.0^{\circ}$, or $1.5^{\circ}$. (B) Rectangular stimulus of Experiment 2 . The rectangle was always presented with a disparity of $0.5^{\circ}$. There was a circular window (diameter, $1.8^{\circ}$ ) in the center. (In order to make the window clearly visible, the rectangle is not drawn to scale.) In the window, a fixation dot was presented with three possible disparities. The disparity of the fixation dot was $0^{\circ}, 0.5^{\circ}$, or $1^{\circ}$. The diameter of the fixation dot was $0.3^{\circ}$. (C) Stimulus used in Experiment 3 . Whenever the occluding foreground bar in front of the rectangle was presented, its horizontal size was $1.6^{\circ}$ and its vertical size $30^{\circ}$. The disparity of the foreground bar was always $1.2^{\circ}$. The disparity of the rectangle was either $0.2^{\circ}, 0.6^{\circ}$, or $1.0^{\circ}$. The foreground bar always contained the monocularly visible contour around it.

means that the different depth planes were monocularly indistinguishable. The rectangular stimuli were always presented in the center of the screen. In particular trials, the rectangles were surrounded by a contour (to make them monocularly visible) and/or accompanied by a planar frontoparallel background (so that the rectangle acted as an occluder). Whenever the background was visible, its size was $60^{\circ} \times 60^{\circ}$. Whenever the contour was visible, it was 6 arcmin wide.

The subjects were instructed to decide whether the vertical dimension of the rectangle was larger than the horizontal dimension. The horizontal dimension of the rectangle was always $5.2^{\circ} ;{ }^{6}$ its vertical dimension varied across trials. The vertical/horizontal aspect ratio of the rectangular stimulus differed between $86 \%$ and $114 \%$. Experiment 1 was divided into two subexperiments, denoted by $1 \mathrm{~A}$ and $1 \mathrm{~B}$.

In Experiment 1A, we were interested in the influence of the disparity on the discrimination task; the disparity between the halfimages was $0.5^{\circ}, 1.0^{\circ}$, or as large as $1.5^{\circ}$. Pilot experiments showed that subjects had no difficulty in judging the aspect ratios of rectangles with a disparity of $1.5^{\circ}$. Regan and Hamstra (1994) showed systematically that aspect ratio discrimination thresholds were not negatively affected by large disparities of up to $2^{\circ}$. The larger the disparity, the clearer the predicted aspect ratio distortion effects. In the introduction, we explained that the predicted distortion effect equals

$$
\frac{S-\delta}{S},
$$

where $S$ is the horizontal dimension of the rectangle and $\delta$ is the relative disparity between the fixation depth and the background; if the alignment-enclosed dimension determines the perceived horizontal dimension of the rectangle, a disparity of $0.5^{\circ}$ would cause a distortion effect of $9.6 \%$. According to a similar calculation, disparities of $1^{\circ}$ and $1.5^{\circ}$ would cause distortion effects as large as $19.2 \%$ and $28.8 \%$, respectively. In Experiment $1 \mathrm{~A}$ (and in the other experiments reported in this paper), the display duration was $2.0 \mathrm{sec}$.

In Experiment 1B, we did a control experiment in which we checked for the influence of the display duration. In Experiment 1B, the display durations were $0.5,2.0$, or $3.5 \mathrm{sec}$. Eye movements were free, and the subjects were encouraged not to perform the task by strict fixation. For a display duration of $0.5 \mathrm{sec}$, the subject would be unable to scan the outline of the complete rectangle. But for a display duration of $3.5 \mathrm{sec}$, the subject would be able to scan the complete outline. Four subjects participated in Experiment 1B. The disparity of the rectangle was $1^{\circ}$.

In both Experiment $1 \mathrm{~A}$ and Experiment $1 \mathrm{~B}$, there were three different stimulus conditions: (1) with a background and with a monocularly visible contour around the rectangle; (2) with a background and without a monocularly visible contour; and (3) without a background. We selected these conditions in order to investigate the difference in performance between purely stereoscopic perception (when the rectangle is invisible monocularly) and perception with monocularly visible information. The three conditions were presented in three individual experimental sessions.

In Experiment 1A, the subject performed 1,188 aspect ratio judgments deriving from three experimental sessions, three conditions (three disparities in Experiment 1A; three display durations in Experiment $1 \mathrm{~B}$ ), eleven aspect ratios, and twelve repetitions per trial. In Experiment 1B, there were 264 fewer trials than in Experiment $1 \mathrm{~A}$, because we did not do the control experiment for all the conditions. We fitted psychometric functions to the data in order to determine the points of perceptual equality $(\mu)$-that is, the aspect ratios that the subjects judged as corresponding to squares.

\section{Results}

Figure 6A shows the results of Experiment $1 \mathrm{~A}$. The rectangle disparity did not have a marked influence on the performance. Ratios obtained in the presence of both a contour and a background were consistently larger than ratios obtained in the other conditions. The subjects reported difficulties in doing the task in the case of a rectangle disparity of $0.5^{\circ}$ when there was a background but no contour around the rectangle (leftmost black diamond 


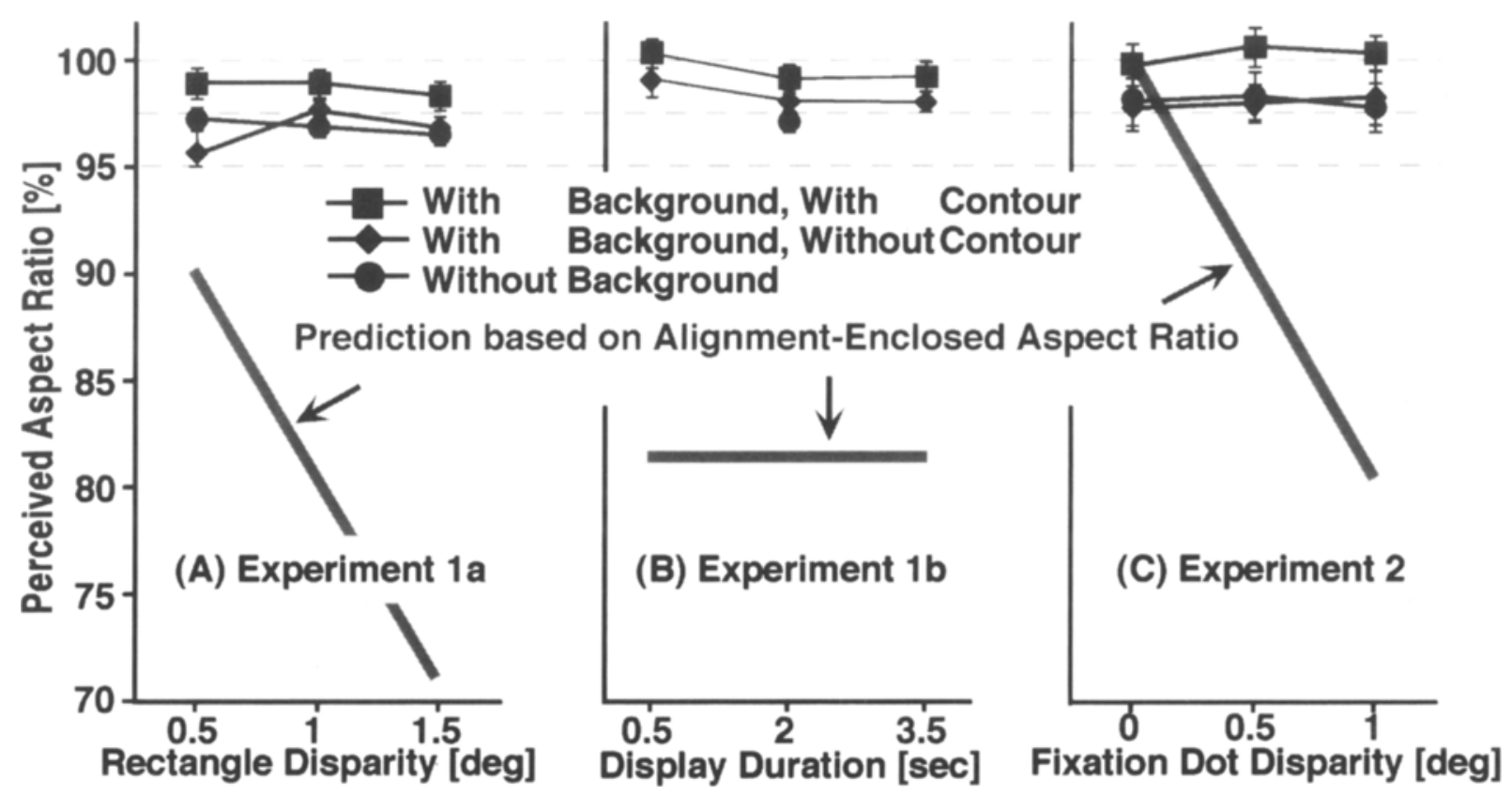

Figure 6. Results of Experiments 1 and 2. The abcissa shows the perceived horizontal/vertical aspect ratio; the ordinate shows the various conditions. For example, a perceived aspect ratio of $95 \%$ means that the subject perceived a rectangle, the vertical dimension of which was smaller $\left(95 \%\right.$ of $\left.5.2^{\circ}\right)$ than the horizontal dimension, as a square. The gray lines depict predictions based on the alignment-enclosed aspect ratio (see Equation 2 and the corresponding text for details). Apart from the fusion problem (see the text) in Experiment $1 \mathrm{~A}$ (panel $\mathrm{A}$, black diamond at $0.5^{\circ}$ ), the rectangle disparity had no significant effect on subjects' performances. In Experiments $1 B$ (panel B) and 2 (panel C), there was no significant effect of the display duration and the fixation depth, respectively. In Experiment $1 B$, no data have been collected for the without-background condition at a display duration of 0.5 and 3.5 sec. Note that, in Experiment 2, in the case in which both a background and a contour were present, there was no horizontal/vertical size illusion. Error bars represent the standard deviation of the data across 8 subjects.

in panel A of Figure 6). They reported that it was difficult to fuse the rectangle at this small disparity; it was not always clear to the subjects which dots at the leftmost and rightmost parts of the rectangle's rim belonged to the background and which to the foreground. Experienced subjects reported that complete correspondence was not accomplished within the display duration. We attribute subjects' significantly different performance in this case, including the large error bar, to a fusion problem. (In Experiment 3, we encountered a similar situation. See Figure 7B for a demonstration of a similarly difficult stimulus.)

Figure 6B shows that performance is veridical for short display durations. The results of Experiments $1 \mathrm{~A}$ and $1 \mathrm{~B}$ are consistent with each other: The values in Figure 6A at a disparity of $1^{\circ}$ should be similar to the values of Figure $6 \mathrm{~B}$ at a display duration of $2 \mathrm{sec}$ because, in Experiment $1 \mathrm{~A}$, we used a display duration of $2 \mathrm{sec}$ and, in Experiment $1 \mathrm{~B}$, we used a square disparity of $1^{\circ}$. Within one standard deviation, the two values were similar.

In all the conditions (except for the shortest presentation duration) of Experiments $1 \mathrm{~A}$ and $\mathrm{BB}$, the subjects perceived the rectangular stimuli as elongated in the vertical direction. We attribute this to the well-known horizontal/ vertical illusion (HVI; see the Discussion section).

\section{EXPERIMENT 2 \\ Influence of the Fixation Depth on Perceived Aspect Ratio}

In normal situations when different depth planes are present, observers probably change their fixation between the different depth planes when judging aspect ratio. The depth of the fixation point is an interesting variable, because it can have a significant influence on perceived aspect ratio (Figure 2). We investigated this possibility in this experiment.

\section{Method}

Experiment 2 was essentially identical to Experiment $1 \mathrm{~A}$. The difference is the fact that fixation in various depth planes was required. Figure 5B shows a schematic drawing of the rectangular stimulus. The rectangle was always presented with a disparity of $0.5^{\circ}$. There was a circular window (diameter, $1.8^{\circ}$ ) in the center of the rectangle. In the window, a fixation dot was presented with three possible disparities (see Figure 7 for a schematic example). The disparity of the fixation dot was $0^{\circ}, 0.5^{\circ}$, or $1^{\circ}$ (which means that the distortions predicted on the basis of the alignment-enclosed aspect ratio were $0 \%, 9.6 \%$, or $19.2 \%$, respectively). The diameter of the fixation dot was $0.3^{\circ}$. In order to minimize eye movements that would be conducted to search for the depth of the fixation dot during the onset of the rectangle, the fixation dot had already been shown for $1 \mathrm{sec}$ prior to the presentation of the stimulus. When 


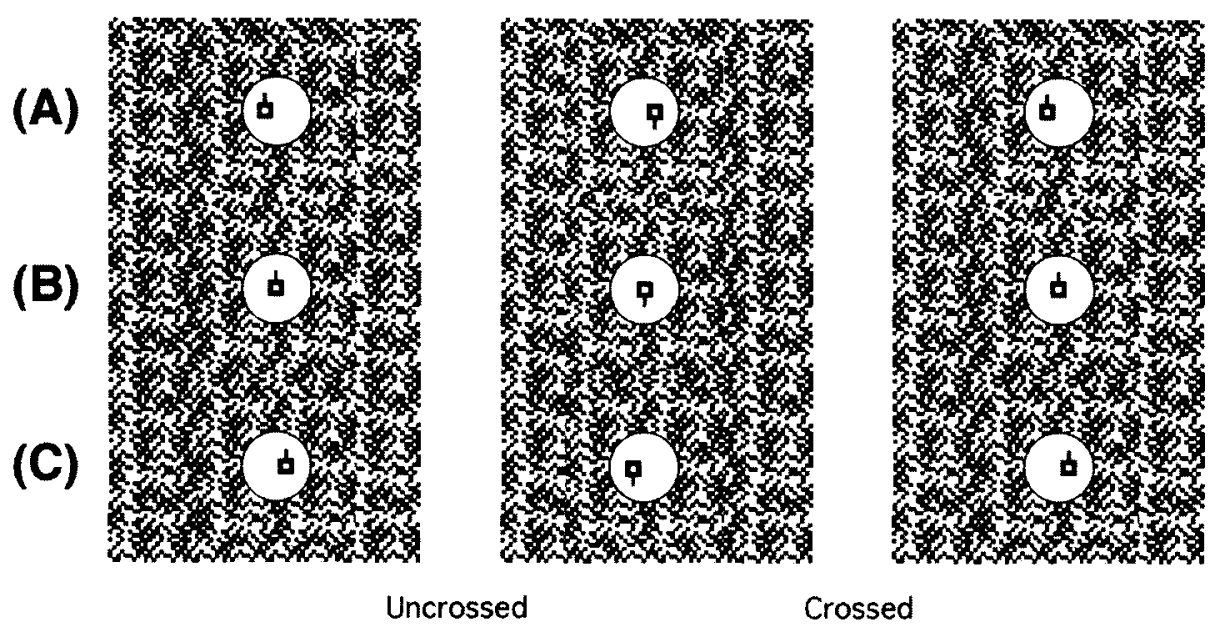

\begin{abstract}
Figure 7. The influence of fixation depth on the perceived aspect ratio of the foreground. Fixation is in the plane of the background (A), in the foreground (B), or in front of the foreground (C). For most observers, there is no significant influence of the fixation depth on the perceived aspect ratio of the foreground rectangle if fusion is established. (Make sure that the viewing distance is large enough for fusion to be established.)
\end{abstract}

strict fixation is required in vision research, stimuli are generally presented with a short duration, such as $75 \mathrm{msec}$, so that completion of a vergence eye movement is impossible. In pilot experiments, however, we found that conducting the task with such a short display duration was not feasible. On the other hand, locked fixation was not really necessary in our experiment because it was sufficient for the subject to keep his or her fixation in one depth plane. Given that the fixation dot had a diameter of $0.3^{\circ}$, it was relatively easy to keep fixation on the dot. We did not measure eye posture during the experiment. On the basis of subjective impressions during preliminary participation in the experiment, we consider it unlikely that occasional unintended vergence eye movements acted as significant contributors to our results. In Experiment 2 (like Experiment $1 \mathrm{~A}$ ), the subjects performed 1,188 aspect ratio judgments.

\section{Results}

Figure 6C shows the results of Experiment 2. The responses did not depend on the disparity of the fixation dot. They were somewhat closer to veridical than were those in Experiment $1 \mathrm{~A}$. This implies that the horizontal dimension of the rectangle was perceived to be somewhat larger (relative to the vertical dimension) than that in Experiment 1. There was no HVI in cases in which both a background and a contour were present. Apparently, the presence of a circular window in conjunction with the presence of a background and a contour was sufficient for a veridically perceived aspect ratio. Although we cannot absolutely rule out the possibility that occasional unintended eye movements contributed to our results, our results provide no support for the hypothesis that fixation depth is important for the stereoscopically perceived aspect ratio of planar surfaces. Figure 7 provides a demonstration of the findings.

\section{EXPERIMENT 3 \\ Perceived Aspect Ratio of an Occluded Pattern}

Now that we knew a number of the essential characteristics of stereoscopic aspect ratio perception in differ- ent depth planes of nonoccluded patterns, we investigated the influence of an occluding bar on the perceived aspect ratio of the rectangle.

\section{Method}

Figure $5 \mathrm{C}$ shows a schematic drawing of the stimulus used in Experiment 3. Except for the presence of the occluding bar, the experiment was similar to Experiment $1 \mathrm{~A}$. The disparity of the rectangle was either $0.2^{\circ}, 0.6^{\circ}$, or $1.0^{\circ}$. The occluder consisted of a vertical bar (width, $1.6^{\circ}$; height, $30^{\circ}$ ). The occluder was always presented in the center of the screen with a disparity of $1.2^{\circ}$. It always contained a surrounding contour in order to facilitate correspondence and fusion. In Experiment 3 we also ran a control condition regarding the influence of eye posture (fixation depth). In this control, we required the subjects to fixate the foreground bar both with and without a contour around the rectangle. We did not calculate predicted distortions based on alignment-enclosed aspect ratio, because there was no tested model for multilayered environments on which such calculations could be based. However, distortions predicted by any model based on alignment-enclosed aspect ratio are similar to the predictions in Experiments 1 and 2.

In Experiment 3, the subject performed 924 aspect ratio judgments. The first experimental series (no fixation, with a contour) consisted of 396 trials: 3 disparities of the rectangle, 11 aspect ratios, and 12 repetitions per trial. Two other experimental series (fixation on the bar) consisted of 264 trials: 2 disparities of the rectangle, 11 aspect ratios, and 12 repetitions per trial.

\section{Results}

Figure 8 shows the results of Experiment 3. Again, our results provide no support for the hypothesis that the fixation depth is important for stereoscopic aspect ratio perception. When a contour was present, the rectangle disparity had no influence on the subjects' performance. As in Experiment 2, we found that the HVI was somewhat weaker in the case of fixation in the plane of the rectangle.

We attribute the value of the open diamond in the left part of Figure 8 to the above-mentioned fusion problem. The open diamond represents the condition in which there 


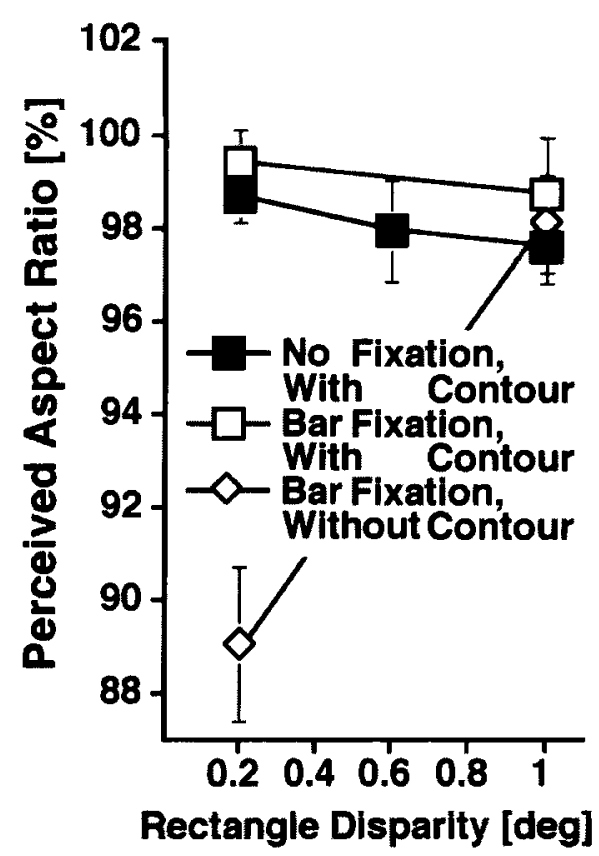

Figure 8. Results of Experiment 3: The mean perceived aspect ratios (vertical/horizontal dimension) as a function of the rectangle disparity. In the with-contour conditions, the rectangle disparity had no significant effect on subjects' performances. The distinguished value of the open diamond at a disparity of $0.2^{\circ}$ is due to the above-mentioned fusion problem (see the text). No data were collected in the bar-fixation/with-contour condition and in the bar-fixation/without-contour (open symbols) condition in the case of a rectangle disparity of $0.6^{\circ}$. Error bars represent the standard deviation of the data across 8 subjects.

was no contour present around the rectangle. In the case of the small rectangle disparity $\left(0.2^{\circ}\right)$, the subjects reported that they found it difficult to fuse the rectangle. Regan and Hamstra (1994) reported a similar finding; they found that discrimination thresholds in aspect ratio judgment of disparity-defined rectangles rose significantly for small disparities. Our subjects reported that it was often not clear which dots at the leftmost and rightmost parts of the rectangle's rim belonged to the background and which to the rectangle. Fusion problems were not reflected merely by increasing standard deviations in the data; they were also reflected by the fact that subjects perceived the horizontal dimension to be significantly smaller than they did when there was fusion. We speculate that this finding reflects a mechanism similar to that found by Erkelens et al. (1996): The location of the rightmost (leftmost) part of the rim is determined by the right (left) eye. ${ }^{7}$ Figure 9 provides a demonstration of the findings.

\section{EXPERIMENT 4 Aspect Ratio of an Occluded and an Unoccluded Pattern}

This experiment was a variation on Experiment 3. In this experiment, we did not investigate the aspect ratio of an occluded pattern (as in Experiment 3), but we studied the perceived aspect ratio of an occluded pattern, relative to a nonoccluded one.

\section{Method}

Figure 10 depicts the stimulus used in Experiment 4. In this experiment, the subjects were instructed to decide whether the (left) rectangle behind the occluder was wider or narrower than the (right) nonoccluded rectangle. The vertical dimensions of the two rectangles were fixed at $4.5^{\circ}$. The horizontal dimensions varied across trials. The horizontal dimension of the right rectangle varied randomly between $86 \%$ and $114 \%$ of the dimension of the left rectangle. There were 11 different aspect ratios presented, which were taken from the following set: $0.86,0.92,0.96,0.98,0.99,1.00,1.01,1.02,1.04$, 1.08 , or 1.14 . The horizontal dimension of the left rectangle was $28.0^{\circ}, 29.8^{\circ}$, or $31.6^{\circ}$. This dimension was varied randomly within a series in order to force the subjects to compare the dimensions of the two rectangles, rather than using some standard dimension stored in memory.

The center of the left (right) rectangle was always presented $18.5^{\circ}$ to the left- (right-) hand side of the center of the screen. Whenever the vertical foreground bar was present, it was presented $18.5^{\circ}$ to the left-hand side of the screen (that is, in the middle of the left rectangle). This was done in order to provide an identical frame of reference for both rectangles so that there would be identical cues providing information about the distance of the rectangle, relative to the foreground. The horizontal dimension of the foreground bar was $1.6^{\circ}$, the vertical dimension $8^{\circ}$. The bar was part of a foreground pattern that was $60^{\circ}$ wide. As in Figure 5, the stimuli consisted of identical random-dot textures (dot size, $3 \times 3$ arcmin). No background was visible.

Experiment 4 consisted of three conditions. In Condition A, there was no occluding bar. This condition was examined in order to check whether the subjects were able to perform the task veridically. In this condition, we did not expect any perceived aspect ratio difference between the left and the right rectangle. In Condition B, the occluding bar was present, but the disparity of the bar was identical to the disparity of the rectangle $\left(0^{\circ}\right)$; there was no difference in depth. In Condition $\mathrm{C}$, the occluding bar was presented in front of the left rectangle. The disparity of the foreground pattern was $1.2^{\circ}$ (crossed). In Experiment 4, the subjects again performed 1,188 judgments. The three conditions were examined in three individual sessions.

\section{Results}

Figure 11 shows the results of Experiment 4. In the case in which the bar was absent (Condition A), the subjects could do the task veridically, with a small standard deviation. In the case in which the bar was present in the same plane as the rectangles (Condition B), the subjects still could do the task veridically, but the standard deviation was somewhat larger. Similar standard deviations occurred when the bar was present in front of the left rectangle (Condition $C$ ). The standard deviations in both Conditions $\mathrm{B}$ and $\mathrm{C}$ are comparable to the standard deviations in Experiments 1-3. In Condition C, the subjects perceived the occluded rectangle to be $1 \%$ smaller than the nonoccluded rectangle. The latter result is consistent with the results of Experiments 1-3.

\section{GENERAL DISCUSSION}

We have presented the results of four psychophysical experiments on a fundamental and theoretically problem- 


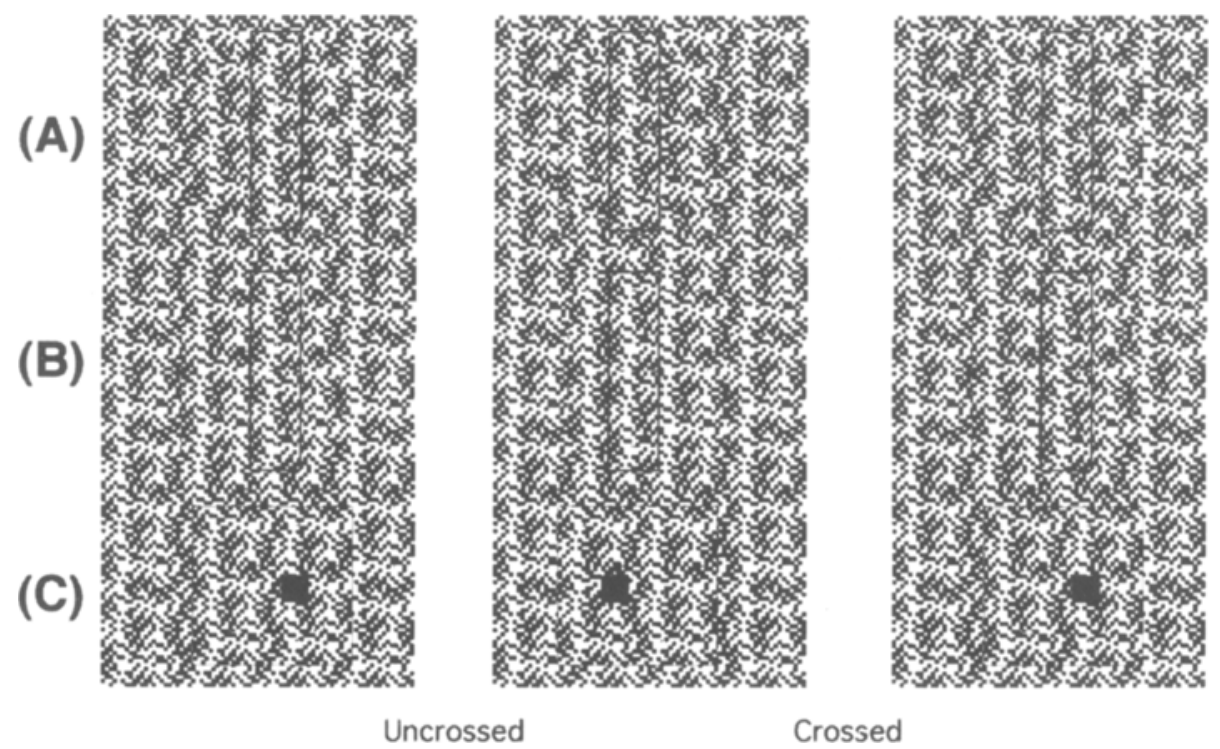

\begin{abstract}
Figure 9. Lack of fusion causes horizontal compression of perceived aspect ratio. (A) The rectangle has such a disparity that it is relatively easy to fuse when observers fixate on the foreground bar. This example illustrates the $1^{\circ}$ disparity condition in Experiment 3. (B) The disparity of the rectangle, relative to the background, is so small that it is hardly fusible when fixation is in the plane of the foreground bar. Lack of fusion causes the square to be perceived as a rectangle, the vertical dimension of which is larger than the horizontal dimension. This example illustrates the $0.2^{\circ}$ disparity condition in Experiment 3 in the absence of the surrounding contour. (C) Fixation should be on the black symbol in front of the rectangle. The disparity of this symbol is large. As in panel $B$, lack of fusion causes the square to be perceived as a rectangle, the vertical dimension of which is larger than the horizontal dimension. The effect is most pronounced when the viewing distance is short, so disparities are relatively large. This example represents most of the conditions in the experiment performed by Liu and Kennedy (see the Discussion section).
\end{abstract}

atic aspect of stereoscopic vision: the interaction of perceived direction and perceived aspect ratio. We have examined the perceived aspect ratio of occluders and partly occluded planes. The standard model of binocular space perception assumes that we view the world from a single vantage point (the so-called cyclopean eye) that is located halfway between the two eyes. In the cyclopean eye, both binocular and monocular elements are represented. One straightforward way to judge the aspect ratio of an object would be to compare the perceived directions of the edges of the object (from the cyclopean eye). In this paper, we have explained that, if an observer were to follow this comparison strategy, perceived aspect ratios of objects located outside the plane of fixation would be perceived in a distorted fashion.

Distortion of objects, as based on the comparison strategy, was not found. The results of Experiments 1 and 2 showed that the observers perceived an occluder to be almost undistorted (in accordance with the subjective results on perceived aspect ratio reported by Erkelens et al., 1996). The results of Experiment 2 provided no support for the hypothesis that fixation depth is important for stereoscopically perceived aspect ratio. The results of Experiments 3 and 4 revealed that the observers perceived the aspect ratio of a partly occluded plane almost veridically. We use the word almost because aspect ratio percep- tion was not completely veridical; there was a consistent overall biasing effect that caused the observers to perceive a square to be slightly (about $2 \%$ ) smaller in the horizontal direction than in the vertical direction. We attribute this finding to the well-known HVI. This will be discussed below. Consider first a summary of our findings.

1. Planar objects were stereoscopically perceived as being undistorted both in and outside the plane of fixation.

2. In Figure 3, we illustrate the fact that all the monocularly visible elements of the visual scene were also represented in the stereoscopic percept of the scene (cf. Erkelens et al., 1996; Erkelens \& van Ee, 1997a, 1997b; Lillakas et al., 1998; Ohtsuka \& Ono, 1998).

3 . Figure 3 also shows that the left/right ordering of details in the stereoscopic domain agreed with this ordering in the two monocular domains (cf. Erkelens et al., 1996; Erkelens \& van de Grind, 1994; Erkelens \& van Ee, 1997a, 1997b; Lillakas et al., 1998; van Ee et al., 1999).

4. Not only the object as a whole, but also the elements of which the object was made, such as the chessboard elements in Figure 3, were perceived to be undistorted.

The inevitable conclusion is that perceived aspect ratio and alignment of visual directions cannot be represented correctly and simultaneously in a 2-D cyclopean eye, just as it is impossible to paint a 3-D picture on canvas. We conclude that perceived aspect ratio does not fol- 


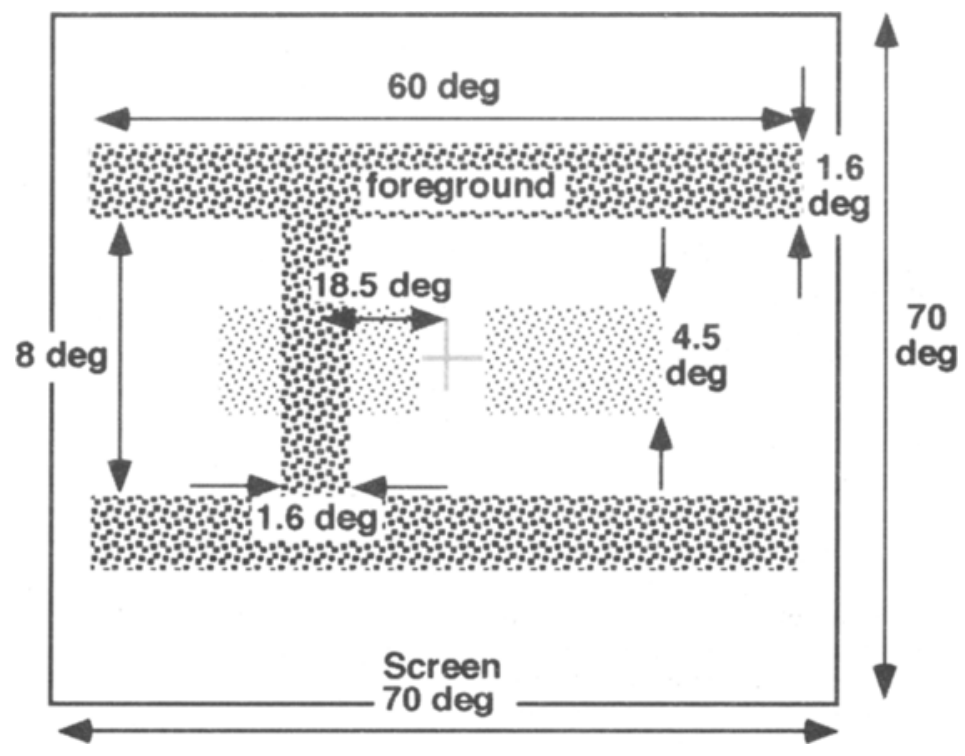

\begin{abstract}
Figure 10. Stimulus of Experiment 4. The vertical dimension of the rectangles was fixed at $4.5^{\circ}$. The horizontal dimension of the left rectangle was $28.0^{\circ}$, $29.8^{\circ}$, or $31.6^{\circ}$. The horizontal dimension of the right rectangle varied randomly between $86 \%$ and $114 \%$ of the dimension of the left rectangle. The subjects were instructed to discriminate whether the rectangle behind the occluder was wider and narrower than the nonoccluded rectangle. The center of the left (right) rectangle was always presented $18.5^{\circ}$ to the left- (right-) hand side of the center of the screen (marked by the gray cross). Whenever the vertical foreground bar (size, $1.6^{\circ} \times 8^{\circ}$ ) was present, it was presented $18.5^{\circ}$ to the left-hand side of the screen (that is, on top of the center of the left rectangle). The bar was part of a foreground pattern that was $60^{\circ}$ wide. The boundaries of the screen (size, $70^{\circ} \times 7^{\circ}$ ) and the cross in the center were not visible. Although the figure shows different textures, the textures in the experiment were identical (Julesz stereogram). There was no background present.
\end{abstract}

low directly from the comparison of visual directions. The interaction of perceived visual directions and perceived shape is a fundamental problem in any model that makes use of a 2-D cyclopean eye.

In the following subsections, we will use demonstrations, as well as the Kanizsa and Poggendorff illusions, to support the notion that the mechanism for visual direction perception and the mechanism for aspect ratio perception might be dissociated and that they cannot be represented simultaneously in 2-D space. We will show that visual space is not distorted locally and that different spatially separated objects do not distort each other with respect to both perceived direction and aspect ratio.

\section{Locality in the Perception of Distorted Aspect Ratio}

It might be that a possible perceived aspect ratio distortion effect is a local effect; if the width of the occluder is only slightly less than the width of the partly occluded object, aspect ratio distortion might be more clearly measurable than we found it to be in our experiments.

Figure 12A gives a demonstration of an occluder that occludes an object of similar size. Although most observers perceive a larger number of chessboard elements in the horizontal direction ( $7 \%$ more, as in Figure 3 ) than in the vertical direction, most observers do not observe such a large aspect ratio distortion. Thus, this demonstration does not support the notion that a perceived aspect ratio distortion effect might be a local effect.

As a matter of fact, this demonstration (Figure 12A) is the 3-D analogy of Kanizsa's compressed square illusion (Kanizsa, 1972), such as is shown in Figure 4. Figure 4A showed that an occluded square in a 2-D drawing looks much more compressed than does a nonoccluded square of the same size. Although a perceived compression of the Kanizsa square is present in a 2-D drawing (Figure 4A), it is absent in stereoscopic viewing when distinct depth planes are involved (Figure 12A; see also Ohtsuka \& Ono, 1998).

In Figure 4A the edges of both the occluded and the nonoccluded squares look as if they are aligned (their perceived visual directions are the same). This is true not only for the original Kanisza figure, but also for one that is rotated over $90^{\circ}$. But the aspect ratios of both the occluded and the nonoccluded squares look very different. So the dissociation between perceived visual directions and perceived aspect ratio that we have identified in 3-D figures also occurs in 2-D ones. 


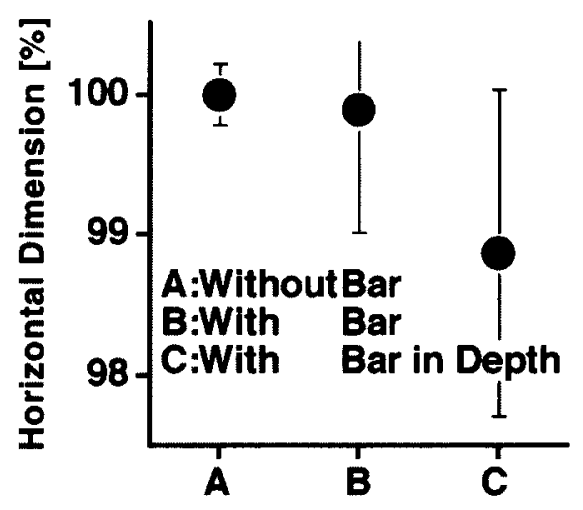

Figure 11. Results of Experiment 4. Horizontal dimension of the left rectangle as a percentage of the horizontal dimension of the right rectangle (for which the subjects found the horizontal sizes equally large) is shown for three conditions. Error bars represent the standard deviation of the data across eight subjects. Condition A, without a bar; Condition B, with a bar, bar disparity of $0^{\circ}$; Condition $C$, with a bar, bar disparity was $1.2^{\circ}$.

\section{Poggendorff Illusion}

A similar dissociation between perceived visual directions and aspect ratio occurs in the Poggendorff illusion. The two lines that are present on either side of the rectangle in Figures 4B and 12B are, in fact, segments of one line. In nonstereoscopic viewing, the intersection of the right line with the occluding bar looks as if it is shifted upward, relative to the intersection of the left line with the bar (Figure 4B). Figure 12B shows that in stereoscopic viewing (given that fusion is established), a similar stimulus does not give rise to this compelling perceptual shift when there is a distinct depth between the rectangle and the oblique line (Drobnis \& Lawson, 1976; Gyoba, 1978; Julesz, 1971). ${ }^{8}$

We recall that in the studies of Erkelens et al. (1996) and Ohtsuka and Ono (1998), 3-D direction perception was well explained by the hypothesis that alignment is based on equating local signs in the eye that is able to see the monocular region caused by the occluder; at the leftmost (rightmost) part of the rim, observers were aligning the slider as if they were aligning it from the left (right) eye (Figures 1 and 3). This idea can be applied to stereoscopic perception of a Poggendorff figure. The fused image can be thought of as being built up of two parts. The left part of the fused image is produced by the left part of the left eye's retinal image. The right part of this fused image is produced by the right part of the right eye's halfimage.

It has not yet been explained why there is a Poggendorff illusion in 2-D figures. Ohtsuka and Ono (1998) proposed that the Poggendorff illusion (and also the Kanizsa illusion) is explained by a mechanism that, perceptually, both displaces and compresses a portion of the visual field in the horizontal direction. (Indeed, this would be a solution that fits all visual directions into a 2-D cyclopean eye.) However, Figure 4 suggests that both illu- sions are essentially 2-D phenomena. If the illusions were caused by horizontal compression of partially occluded depth planes, they should vanish whenever the occluder is rotated by $90^{\circ}$. Figure 4 shows that the illusions persist and are basically related to the orientation of the occluding rectangle. Explanations of the 2-D Kanizsa and Poggendorff illusions are tangential to this paper, and we will not go into further detail. We will show, however, that there is no perceived displacement or compression visible in 3-D stereoscopic viewing when distinct depth planes are involved. Figure 13 illustrates an example in which there are three occluders in front of a background that consists of three spatially separated chessboard patterns. After fusion, you will see that all the information that is monocularly visible is also present in the stereoscopic percept; there is no distortion. Neither is there any distortion (shift or compression) visible in the perceived aspect ratio of the background chessboard elements. Nor is a shift visible in the domain of visual directions. And there is no distortion of perceived foreground aspect ratio either. The three separated objects do not influence the perceived spatial properties. More generally, it is not clear how a compensation mechanism that, perceptually, both displaces and compresses a portion of the visual field (Ohtsuka \& Ono, 1998) works for planes that are slanted, relative to the head. Intuitively, we expect that only a 3-D representation of visual space contains the information that is essential for the correct estimation of the aspect ratio of slanted planes. Slanted planes form a potentially interesting stimulus with which to test the representation of aspect ratio in 3-D space.

\section{Horizontal/Vertical Illusion}

A comment about the HVI is in order because we found, on average, an HVI of about $2 \%$. Our results enable us to evaluate some of the explanations for the HVI provided in the literature.

We restrict this discussion to the HVI in rectangles. ${ }^{9}$ Fick (1852) is usually given credit for first drawing attention to the HVI. Fick reported that a bright square on a dark ground looks like a vertical oblong. There have been very few studies on the quantitative magnitude of the effect in rectangular features, and results across authors differ considerably. Fick (1852) presented quantitative results, although for 1 observer only. He found at two distances, on average, an effect of 7\%. Veniar (1948) determined thresholds of the effect for 5 subjects and found, on average, a distortion of $1.4 \%$. There are also reports of authors who did not find the illusion (e.g., Sleight \& Austin, 1952). The illusion has been investigated in artificial fields (Künnapas, 1959), in rich visual environments (Chapanis \& Mankin, 1967), and haptically (Tedford \& Tudor, 1969). As far as we know, the illusion has not been investigated in the stereoscopic domain. The explanations of the illusion to be found in the literature (for a review, see Higashiyama, 1996) can be divided into four groups: gravity, physiology, perspective, and frame of reference. ${ }^{10}$ The first two explanations have no bearing 
(A) Kanizsa Figure

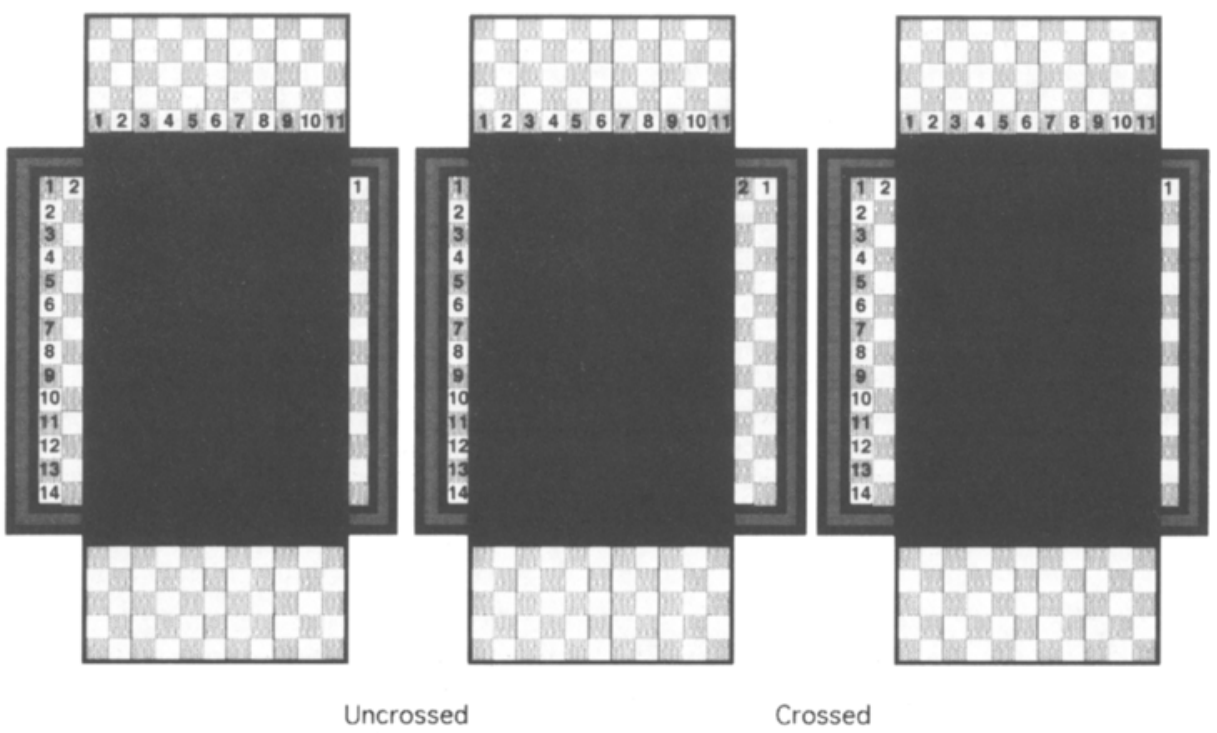

(B) Poggendorff Illusion

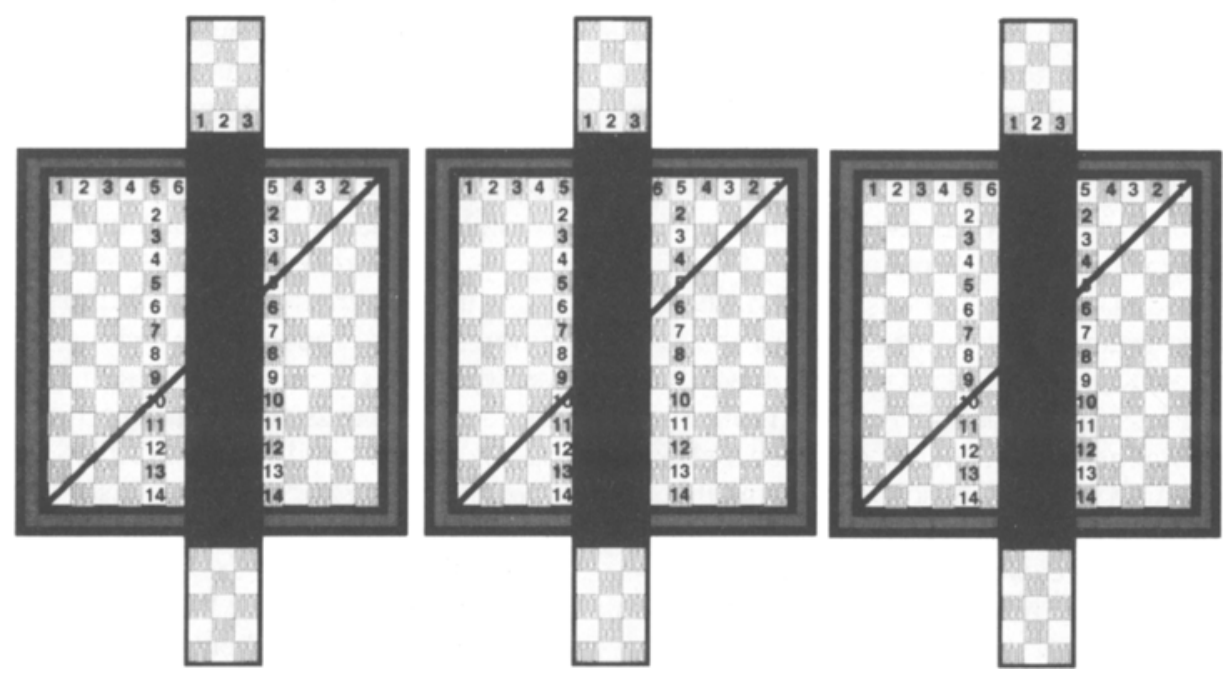

Figure 12. The Kanizsa and Poggendorff illusions in stereoscopic viewing. (A) A Kanizsa figure with distinct depth planes in stereoscopic viewing does not give rise to the compelling shrinkage that we saw in the 2-D drawing in Figure 4A. After fusion of the two half-images, the square in the background looks square or only slightly compressed in the horizontal direction, relative to the vertical direction (due to the horizontal/vertical size illusion). (B) A Poggendorff figure with distinct depth planes in stereoscopic viewing does not give rise to the compelling perceptual shift that we saw in Figure $4 \mathrm{~B}$. In the fused image, it looks as if there is one straight line in the plane of the square that is occluded by the rectangle. The feature behind the bar looks square (or slightly elongated in the vertical direction, owing to the horizontal/vertical size illusion).

on our paper, and we will disregard them. The perspective explanation states that a horizontal line in the picture plane does not indicate depth, but a vertical line frequently depicts a line receding into depth. Such a perspective cue evokes inappropriate size constancy scaling (Gregory, 1963). According to the size constancy theory, objects that are farther away should be larger; this explains why the vertical line is perceived as being larger than the horizontal line that is perceived as being in the picture plane.
Our results suggest that it is not likely that this perspective explanation is the sole reason for the illusion. In our set-up, the subjects perceived the square clearly at a constant depth plane in front of the screen. There is no perspective cue that evokes perception of the verticals' receding in depth. However, the illusion is still present.

Künnapas explained the HVI within the context of a frame-of-reference theory (for a review, see Higashiyama, 1996, or Prinzmetal \& Gettleman, 1993). The nearer a 


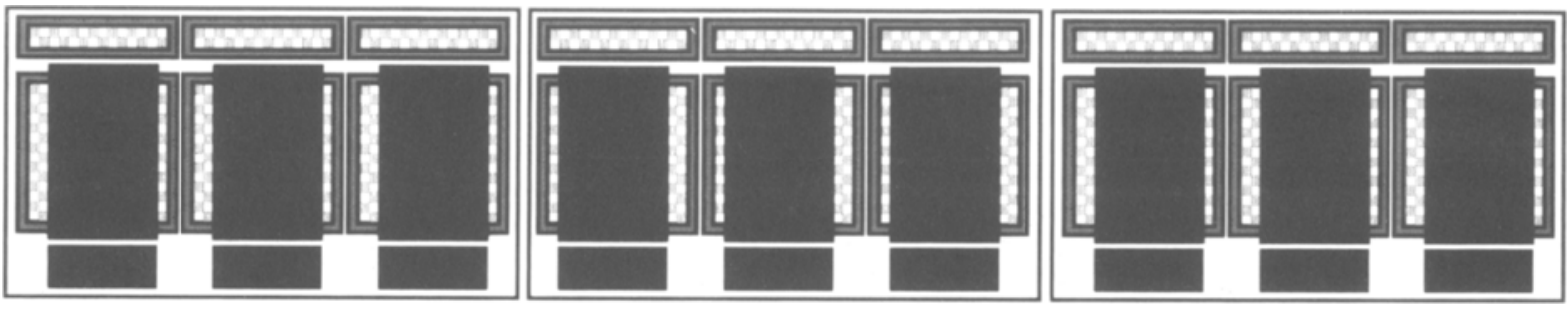

Uncrossed

Crossed

Figure 13. No perceived distortion of direction or aspect ratio in structured space. Observers perceive no local aspect ratio distortion when three Kanizsa squares are presented next to each other. The stimuli above and below the central figures are for reference. In monocular viewing, the chessboard patterns seem to be compressed in the horizontal direction. The references show that the borders of both the occluder and the chessboards do not seem to be displaced (as in Figure $4 \mathrm{~A}$ ), either relative to the reference objects or relative to its neighbors. This observation supports the presence of a dissociation between perceived direction and aspect ratio. Note that all monocular information is visible in the stereoscopic percept and that the left/right ordering in the stereoscopic percept corresponds to the left/right ordering in the monocular views.

line is to a surrounding frame, the longer it appears to be (Künnapas, 1955). Because the stereoscopic visual field is horizontally elongated relative to the vertical dimension, objects will generally be closer to the boundary of the visual field than horizontal lines will be, and hence, vertical lines will appear to be longer. This explanation considers the HVI as a 2-D interaction. Like the Poggendorff and the Kanizsa illusion, it does not depend on the depth within the scene. Künnapas' (1955) explanation is consistent with the findings in this paper. We did not find that the disparity influenced the magnitude of the illusion. But we found that the illusion was reduced when the circular window was present in the center of the foreground rectangles. Künnapas (1955) predicted that the illusion would be less in monocular viewing (which was confirmed by the experimental results obtained by Prinzmetal \& Gettleman, 1993). In our study, we found that the illusion was reduced when a monocularly visible contour surrounded the rectangle, which was otherwise monocularly invisible. Collectively, we found no evidence against the framing theory.

It is worth noting that none of the explanations of the HVI is related either to the local signs as detected by the visual system or to the interaction of perceived visual directions and perceived aspect ratio. Therefore, the interpretations of the data are not influenced by the illusion.

\section{Related Work on Perceived Aspect Ratio}

Ohtsuka and Ono (1998) performed measurements on the perceived aspect ratio of a partly occluded square. Their experiment is comparable with our Experiment 4, Condition $\mathrm{C}$ (although they did stereoscopic viewing, their stimuli were monocularly visible). For a Kanizsa feature (Figure 12A) that was presented as a stereogram, they found that the occluded square was perceived as being slightly compressed horizontally (HVI), relative to a nonoccluded square. Their results in this condition are identical to our findings in Experiment 4.

Regan and Hamstra $(1991,1992,1994)$ conducted extensive studies on the perceived aspect ratio of rectangles defined in many different ways, including by disparity. In most of their experiments, the subjects' vision was adapted to rectangles with a certain aspect ratio before they made their decisions. Except for one remark in their 1992 paper, Regan and Hamstra do not report on the precision of aspect ratio judgment for a nonadapted isolated rectangle. According to this one remark, the subjects showed significantly more veridical behavior in judging squareness than did our subjects, and they did not show the HVI. It should be noted, however, that their subjects were trained in judging squareness by using feedback.

Liu and Kennedy (1995) found a clear horizontal compression of occluded rectangles, depending on fixation depth. This means that our findings seem to be at odds with their findings. They also found - in apparent contrast to Drobnis and Lawson (1976), Julesz (1971), and others-that the Poggendorff illusion was clearly present in the stereoscopic percept. They claimed that the fixation depth determined the strength of the misalignment of the oblique line in the Poggendorff illusion. However, closer examination of their methods reveals that they presented double images caused by unfusible large disparities. ${ }^{11}$ In the case of unfused images (Figure 9 or note 6), we also found compression of the occluder. In their model, they employed both the induced misalignment caused by this compression and the fact that only one of the halfimages was visible (because the other one was suppressed by rivalry) in order to explain the Poggendorff illusion in stereoscopic viewing. It is hard to validate their explanation, because they did not make their model explicit. That is, they did not incorporate the vertical location of the intersection of the line and the occluder on either side of the occluder. They did not relate their model to visual directions either. Although, unfortunately, their data and model merely cover unfused images, both their data and their model are not in contradiction with the present findings.

\section{Conclusion}

In stereoscopic vision research, we are interested in how we perceive the 3-D layout of a scene. Measuring per- 
ceived horizontal/vertical aspect ratios is a useful tool for assessing to what extent models of space perception are applicable to perception in day-to-day circumstances with frequently occurring occlusions. Our results, the demonstrations in this paper, as well as the evidence provided by other literature, leads to the following conclusions regarding the interaction of perceived direction and aspect ratio. (1) The perceived aspect ratio and visual directions cannot be correctly represented simultaneously in a 2-D cyclopean eye. (2) The perceived aspect ratio in 2-D and 3 -D stimulus configurations does not follow from the direct comparison of perceived visual directions. (3) Except for the HVI, our data do not provide any evidence that the perceived aspect ratio of planar objects (both occluders and occlusions) is distorted, either in the plane of fixation or outside this plane. (4) Not only the object as a whole, but also the elements of which the object consists are perceived to be undistorted. (5) All monocularly visible parts of the visual scene are also represented in the stereoscopic percept of the scene. (6) The left/right ordering of details in the stereoscopic domain agrees with this ordering in the two monocular domains. (7) Both the Poggendorff and the Kanizsa illusions are 2-D phenomena. They are not present in stereoscopic vision when distinct depth planes are involved. (8) We suggest that the HVI is also a 2-D phenomenon. It does not depend on the depth of the object, relative to the background. It is suggested that the visual environment is the inducer of the illusion, which supports the framing theory of Künnapas.

Taken together, the results strongly suggest that the mechanism that determines perceived aspect ratio is dissociated from the mechanism that determines perceived direction. Only a 3-D representation of visual space contains the information that is essential for the correct estimation of the aspect ratio of slanted planes. Slanted planes form a potentially interesting stimulus with which to further investigate the underlying mechanism of stereoscopically perceived aspect ratio.

\section{REFERENCES}

Anderson, B. L., \& Nakayama, K. (1994). Toward a general theory of stereopsis: Binocular matching, occluding contours, and fusion. Psychological Review, 101, 414-445.

Banks, M. S., VAN EE, R., \& BackUS, B. T. (1997). The computation of binocular visual direction: A re-examination of Mansfield and Legge (1996). Vision Research, 37, 1605-1610.

Chapanis, A., \& Mankin, D. A. (1967). The vertical-horizontal illusion in a visually-rich environment. Perception \& Psychophysics, 2 , 249-255.

Drobnis, B. J., \& Lawson. R. B. (1976). The Poggendorff illusion in stereoscopic space. Perception \& Motor Skills, 42, $15-18$.

ERKEl.ens, C. J., MuiJs, A. J. M., \& VAN EE, R. (1996). Binocular alignment in different depth planes. Vision Research, 36, 2141-2147.

Erkelens, C. J., \& van de Grind, W. A. (1994). Binocular visual direction. Vision Research, 34, 2963-2969.

ERKelens, C. J., \& van Ee, R. (1997a). Capture of visual direction: An unexpected phenomenon in binocular vision. Vision Research, 37, 1193-1196.

Erkelens, C. J., \& van Ee, R. (1997b). Capture of visual direction of monocular objects by adjacent binocular objects. Vision Research, $\mathbf{3 7}$, 1735-1745
Fick, A. (1852). Erörterung eines physiologisch-optischen phänomens. Zeitschrift für rationelle Medizin, 2, 83-94.

FINGER, F. W., \& SPELT, D. K. (1947). The illustration of the horizontalvertical illusion. Journal of Experimental Psychology, 37, 243-249.

GoGel, W. C. (1963). Visual perception of size and distance. Vision Research, $3,101-120$.

Gogel, W. C. (1975). Depth adjacency and the Ponzo illusion. Perception \& Psychophysics, 17, 125-132.

GrEGORY, R. L. (1963). Distortion of visual space as inappropriate constancy scaling. Nature, 199, 678-679.

GrobA, J. (1978). The Poggendorff illusion under stereopsis. Tohoku Psychologica Folia, 37, 94-101.

Higashiyama, A. (1996). Horizontal and vertical distance perception: The discorded-orientation theory. Perception \& Psychophysics, $\mathbf{5 8}$, 259-270.

Howard, 1. P.. \& Rogers, B. J. (1995). Binocular vision and stereopsis. New York: Oxford University Press.

JulEsz, B. (1971). Foundations of cyclopean perception. Chicago: University of Chicago Press.

Kanizsa, G. (1972). Schrumpfung von visuellen Feldern bei amodaler Ergänzung. Studia Psychologica, 14, 208-210.

KüNNAPAS, T. M. (1955). Influence of frame size on apparent length of a line. Journal of Experimental Psychology, 50, 168-170.

KünNaPAS, T. M. (1959). The vertical-horizontal illusion in artificial fields. Journal of Psychology, 47, 41-48.

Lillakas, L., ONo, H., \& Grove, P. M. (1998). The visual system's solution to Leonardo Da Vinci's paradox when both the occluder and the background are fused [Abstract]. Investigative Ophthalmology \& $V_{i-}$ sual Science, 39, S615.

LiU, C. H., \& KENNEDY, J. M. (1995). Misalignment effects in 3-D versions of Poggendorff displays. Perception \& Psychophysics, 57, 409415.

MaPP, A. P., \& ONO, H. (1999). Wondering about the wandering cyclopean eye. Vision Research, 39, 2381-2386.

Ohtsuka, S., \& ONO, H. (1998). Adjustment for displacement and compression triggered by pictorial cue of occlusion produces correct 3-D perception and Kanizsa's illusion [Abstract]. Investigative Ophthalmology \& Visual Science, 39, S850.

ONO, H., \& MAPP, A. P. (1995). A restatement and modification of Wells-Hering's laws of visual direction. Perception, 24, 237-252.

Prinzmetal, W., \& Gettleman, L. (1993). Vertical-horizontal illusion: One eye is better than two. Perception \& Psychophysics, 53, 81 88.

Regan, D., \& Hamstra. S. J. (1991). Shape discrimination for motiondefined and contrast-defined form: Squareness is special. Perception, 20,315-336.

Regan, D., \& Hamstra, S. J. (1992). Shape discrimination and the judgment of perfect symmetry: Dissociation of shape from size. $\mathrm{Vi}$ sion Research, 32, 1845-1864.

REgan, D., \& Hamstra, S. J. (1994). Shape discrimination for rectangles defined by disparity alone, by disparity plus luminance and by disparity plus motion. Vision Research, 34, 2277-2291.

Schor, C. M. (1999). Binocular vision. In K. Devalois (Ed.), Seeing. Handbook of perception and cognition (2nd ed.). New York: Academic Press.

Sleight, R. B., \& Austin, T. R. (1952). The horizontal-vertical illusion in plane geometric figures. Journal of Psychology, 33, 279-289.

Suzukı, M., Segal, O., Lillakas, L., \& ONo, H. (1998). The visual system's solution to Leonardo Da Vinci's paradox when retinal disparity between the occluder and the background is large [Abstract] Investigative Ophthalmology \& Visual Science, 39, S618.

TEDFORD, W. H., \& TUDOR, L. L. (1969). Tactual and visual illusions in the T-shaped figure. Journal of Experimental Psychology, 81, 199-201

van de Grind, W. A., Erkelens, C. J., \& LaAn, A. C. (1995). Binocular correspondence and visual direction. Perception, 24, 215-235.

van Ee, R., Banks, M. S., \& Backus, B. T. (1999). Perceived visual direction near an occluder. Vision Research, 39, 4085-4097.

VAN Ee, R., \& ERKEl.ens, C. J. (1995). Binocular perception of slant about oblique axes relative to a visual frame of reference. Perception, 24, 299-314. 
VENIAR, F. A. (1948). Difference thresholds for shape distortion of geometrical squares. Journal of Psychology, 26, 461-476.

\section{NOTES}

1. Note that this analysis is essentially independent of both the presence and the location of the cyclopean eye (Figure 2). After completion of this manuscript, Mapp and Ono (1999) stated that the cyclopean eye "is both a logical and a functional necessity for judging the direction of one object with respect to another." In addition, they suggested that the findings about binocular alignment in different depth planes (Erkelens et al., 1996) and about capture of visual directions (Erkelens \& van Ee, 1997a, 1997b) were based on "a wandering cyclopean eye." Their statement and suggestions are not justified: Our analyses were based on visual lines and eye orientations, which means that the location of the cyclopean eye is irrelevant for the validity of the analyses (for the latter claim, see also Banks, van Ee, \& Backus, 1997).

2. Stereograms in this paper consist of three half-images. Observers who have the best fusion when their eyes are crossed (which means that the half-image on the right side is seen by the left eye) should fuse the two half-images on the right side; uncrossed fusers (or observers who use a stereoscope) should fuse the two images on the left. Independent of the eyes' cross-mode, the half-image that is seen by the left eye is called the left eye's half-image; the half-image seen by the right eye, the right eye's half-image.

3. These results, in turn, can be understood within the larger framework of a mechanism that has been called capture of perceived direction (Erkelens \& van Ee, 1997a, 1997b). Van Ee et al. (1999) showed that such a mechanism is at work even if monocular features are not due to a stimulus situation that can be interpreted by the visual system as being caused by occlusion geometry. In this paper, we will disregard the mechanisms underlying the perceived direction of targets.

4. There are slightly different versions of the standard model, but all of them are based on the concept of a 2-D cyclopean eye midway between the two real eyes.

5 . We also determined the slopes of the psychometric curves in all of our experiments. In general, the slopes were between $0.5 \% \pm 0.1 \%$ and $5.5 \% \pm 0.5 \%$, which are similar to the slopes found by Regan and Hamstra (1994) in a discrimination experiment in which subjects judged the aspect ratios of disparity-defined rectangles. In general, the slopes did not differ markedly between conditions for a particular subject (see also Regan \& Hamstra, 1992). In this paper, we do not further analyze these slopes.

6. In a control experiment, we repeated one condition of the experiment while presenting rectangles with two horizontal dimensions-- $5.2^{\circ}$ and $8.0^{\circ}$-intermixed within one series, in order to control for the possibility that the subjects were able to perform the task by comparing the vertical dimension of the rectangle with some internal reference (after a couple of trial blocks, the range of stimuli and, consequently, the vertical dimension that is identical to the horizontal dimension could be known to the subject and stored in memory), rather than by comparing the vertical dimension with the horizontal dimension. Results were not significantly affected by this size variation. Regan and Hamstra (1991, 1992, 1994) explicitly disconfounded perceived aspect ratio from size perception by requiring subjects to discriminate aspect ratios of rectangles whose areas were altered independently of aspect ratio. They found near-perfect behavior, although the subjects could not use the dimension of the rectangle sides.

7. The compression in the horizontal dimension caused by the absence of fusion can be readily observed when one holds one's naked forearm vertically in front of the nose at a distance of $15 \mathrm{~cm}$. The effect is strongest when one fixates in the background.

8. For relative depths that are very small (smaller than 4.5 arcmin; Drobnis \& Lawson, 1976), the Poggendorff illusion is strongly reduced, but it still exists. This result can be interpreted in the context of the adjacency principle of Gogel $(1963,1975)$.

9. Finger and Spelt (1947) showed that the HVI is frequently illustrated with a feature (for instance, a T-figure), whereas in fact, the bisection illusion is responsible for the difference in perceived line length. Often an L-figure is used to study the illusion.

10. In the gravitational explanation, a line parallel with gravity is perceived as larger than a line of the same dimension that lies perpendicular to gravity. In the physiological explanation, eye movements and properties of the neurophysiology of the retina and the brain are thought to contribute to the HVI.

11. The rectangle in Liu and Kennedy's (1995) Poggendorff figure was positioned in front of the eyes at a distance of $15 \mathrm{~cm}$. The oblique line varied in viewing distance from $15 \mathrm{~cm}$ up to $75 \mathrm{~cm}$ in steps of $10 \mathrm{~cm}$. This means that the disparities were about $0^{\circ}, 10^{\circ}, 14^{\circ}, 17^{\circ}, 18^{\circ}, 19^{\circ}$, and $20^{\circ}$. In other words, even their smallest nonzero disparity was larger than binocular fusion allows. And indeed, they noted (p. 413) that it is "only with deliberate effort (that) one observes the presence of the doubled ends of the oblique" (usually the doubled ends of the oblique were suppressed by the rivalry mechanism). From their work it is not entirely clear whether they intended to present their subjects with merely unfusible disparities.

(Manuscript received June 16, 1998; revision accepted for publication June 10, 1999.) 